\title{
Fixed point sets of parabolic isometries of CAT(0)-spaces
}

\author{
Koji Fujiwara, Koichi Nagano and Takashi Shioya*
}

\begin{abstract}
We study the fixed point set in the ideal boundary of a parabolic isometry of a proper CAT(0)-space. We show that the radius of the fixed point set is at most $\pi / 2$, and study its centers. As a consequence, we prove that the set of fixed points is contractible with respect to the Tits topology.
\end{abstract}

Mathematics Subject Classification (2000). 53C20.

Keywords. CAT $(\kappa)$-space, ideal boundary, Tits metric, parabolic isometry.

\section{Contents}

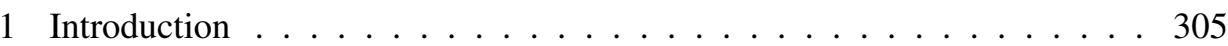

2 Preliminaries . . . . . . . . . . . . . . . . . . 309

3 Estimate of radii of fixed point sets . . . . . . . . . . . . . 311

4 Dimension of Tits ideal boundaries . . . . . . . . . . . . . . 315

5 CAT(1)-spaces of small diameter . . . . . . . . . . . . . . . . 317

6 Example of a symmetric space . . . . . . . . . . . . . . . 323

\section{Introduction}

CAT(0)-spaces are generalizations of Hadamard manifolds in Riemannian geometry to geodesic spaces. The classification of isometries of the hyperbolic plane into elliptic, hyperbolic, and parabolic applies to the CAT(0) setting. The flat torus theorem (cf. $[\mathrm{B}],[\mathrm{BH}]$ ), which is one of the important results concerning hyperbolic isometries, remains true for $\mathrm{CAT}(0)$-spaces.

In the study of isometries of Hadamard manifolds and CAT( 0$)$-spaces, hyperbolic isometries have been extensive. We remark that in a cocompact, proper, isometric

\footnotetext{
*The authors are partially supported by the Grant-in-Aid for scientific research of Japanese Ministry of Education, Culture, Sports, and Technology. The second author is partially supported by the 2004 JSPS Postdoctoral Fellowships for Research Abroad.
} 
group action, there are no parabolic isometries. However, if one does not include the cocompactness assumption, then the group could contain parabolics.

In this paper, we focus on parabolic isometries of CAT(0)-spaces. We generalize the results of Schroeder from Appendix 3 in [BGS] on the fixed point set of a parabolic isometry in the ideal boundary of an Hadamard manifold to the setting of proper CAT(0)-spaces. Our results are not straightforward generalizations since much less has been known concerning analysis on proper CAT(0)-spaces than on Hadamard manifolds. As a new ingredient in our argument, we study the geometry of complete improper CAT(1)-spaces. We then apply the results to the Tits ideal boundary of a CAT(0)-space. At the end, as an example of our theorems, we examine a symmetric space in detail.

1.1. Main theorems and examples. Let $X$ be a complete CAT(0)-space and $X(\infty)$ the ideal boundary of $X$ defined as the asymptotic classes of rays in $X$. We classify an isometry $f$ of $X$ as elliptic, hyperbolic (or axial), or parabolic. $f$ is called elliptic if it has a fixed point in $X$, and hyperbolic if there exists an invariant geodesic line, called axis, $\gamma$ in $X$ such that $f$ acts on $\gamma$ by a non-trivial translation. If $f$ is neither elliptic nor hyperbolic, then it is called parabolic. We recall that $f$ is parabolic if and only if the displacement function $d_{f}(p):=d(p, f(p))$ of $f$ does not attain its infimum in $X . X(\infty)$ is equipped with a natural topology called sphere topology. Any isometry of $X$ also acts as a homeomorphism of $X(\infty)$ since the isometry takes geodesics to geodesics.

It is known that if $X$ is proper (i.e., any closed bounded subset is compact), then any parabolic isometry of $X$ has at least one fixed point in $X(\infty)$ (cf. [B], [BH]). This does not necessarily hold if $X$ is improper. In fact, there is an example of a parabolic isometry $f$ of a separable Hilbert space $X$ of infinite dimension, which is an improper CAT(0)-space, such that $f$ has no fixed point in $X(\infty)$ (and in $X$ ) (cf. [BH]). We denote by $X_{f}(\infty)$ the fixed point set of $f$ in $X(\infty)$.

The ideal boundary $X(\infty)$ has a natural metric, called the Tits metric, denoted Td. The metric space $(X(\infty), T d)$, the Tits ideal boundary, is a complete CAT(1)-space. The topology defined by the Tits metric on $X(\infty)$ is stronger than the sphere topology.

For a metric space $(Y, d)$, we define

$$
\operatorname{rad} Y=\operatorname{rad}(Y, d):=\inf _{x \in Y} \sup _{y \in Y} d(x, y),
$$

which is called the radius of $Y$. For $p \in X$, we denote by $\Sigma_{p} X$ the space of directions at $p$. As one of the main results of this paper, we state the following:

Theorem 1.1. Let $X$ be a proper $\mathrm{CAT}(0)$-space such that $\Sigma_{p} X$ is compact for every $p \in X$, and let $f$ be a parabolic isometry of $X$. Then we have $\operatorname{rad}\left(X_{f}(\infty), T d\right) \leq \pi / 2$. In particular, $\left(X_{f}(\infty), T d\right)$ is contractible. 
Schroeder has proved Theorem 1.1 for smooth Hadamard manifolds in Appendix 3 in [BGS]. We remark that $\pi / 2$ is the optimal upper bound of $\operatorname{rad} X_{f}(\infty)$ even for Hadamard manifolds (cf. Example 1.4). We also have some examples with $0<\operatorname{rad} X_{f}(\infty)<\pi / 2$ (cf. Examples 1.5 and 1.6). Notice that if $X$ is proper and geodesically complete, then $\Sigma_{p} X$ is compact for any $p \in X$.

Recall that $X$ is visible if and only if $T d(x, y)=\infty$ for any distinct points $x, y \in X(\infty)$. By Theorem 1.1, we immediately obtain:

Corollary 1.2. Under the same assumption as in Theorem 1.1, if $X$ is visible, then $X_{f}(\infty)$ consists of a single point.

Buyalo [Bu] has shown that if $X$ is a complete, not necessarily proper, Gromovhyperbolic CAT(0)-space, then the infimum of the displacement function of any parabolic isometry $f$ of $X$ is equal to zero, and $X_{f}(\infty)$ consists of a single point. Let $X$ be a proper CAT(0)-space. If $X$ is Gromov-hyperbolic, then $X$ is visible. If $X$ admits a cocompact group action, then the converse is true (cf. $[\mathrm{BH}]$ ).

Next, we study the centers of $X_{f}(\infty)$. A center of a metric space $(Y, d)$ is a point in $Y$ where the function $Y \ni x \mapsto \sup _{y \in Y} d(x, y) \in[0, \infty]$ attains the infimum, $\operatorname{rad} Y$. We denote by $C(A)$ the set of all centers of $A$, and define $C^{2}(Y):=C(C(Y))$.

Theorem 1.3. Let $X$ be a proper CAT(0)-space of finite covering dimension such that $\Sigma_{p} X$ is compact for every $p \in X$. Let $f$ be a parabolic isometry of $X$. Then $C^{2}\left(X_{f}(\infty)\right)$ consists of a single point, which is fixed by any isometry of $X$ leaving $X_{f}(\infty)$ invariant. In particular, the point is a fixed point of any isometry of $X$ commuting $f$.

Theorem 1.3 for Hadamard manifolds has been shown by Eberlein [E] following Schroeder's work in Appendix 3 in [BGS].

We give some examples.

Example 1.4. Let us denote the hyperbolic plane by $\mathbb{H}^{2}$. We consider the product Riemannian manifold $X:=\mathbb{R} \times \underbrace{\mathbb{H}^{2} \times \cdots \times \mathbb{H}^{2}}_{m \text { times }}, m \geq 1$. For $m$ parabolic isometries $h_{1}, h_{2}, \ldots, h_{m}$ of $\mathbb{H}^{2}$, we define the product map $f:=\left(\mathrm{id}_{\mathbb{R}}, h_{1}, \ldots, h_{m}\right)$, where $\mathrm{id}_{\mathbb{R}}$ is the identity map on $\mathbb{R}$. $f$ is a parabolic isometry of $X$. We denote by $\mathbb{S}^{m-1}(1)$ the standard unit $(m-1)$-sphere in the Euclidean $m$-space $\mathbb{E}^{m}$ and set

$$
\triangle_{1}^{m-1}:=\left\{\left(x_{1}, \ldots, x_{m}\right) \in \mathbb{S}^{m-1}(1) \subset \mathbb{E}^{m} \mid x_{i} \geq 0 \text { for all } i\right\},
$$

which we call the standard spherical $(m-1)$-simplex. $X_{f}(\infty)$ is isometric to the spherical suspension over $\triangle_{1}^{m-1}$. We refer [BBI] for the definition of spherical suspension. We have $\operatorname{rad} X_{f}(\infty)=\pi / 2 . C\left(X_{f}(\infty)\right)$ is isometric to $\triangle_{1}^{m-1}$ and $C^{2}\left(X_{f}(\infty)\right)$ consists of the barycenter of $\triangle_{1}^{m-1}$. 
The following example is discussed in Section 6.

Example 1.5. We consider $X:=\operatorname{SL}(3, \mathbb{R}) / \operatorname{SO}(3)$, which is a five-dimensional, irreducible symmetric space of non-compact type and rank two. SL $(3, \mathbb{R})$ is the identity component of the isometry group of $X$. The Tits ideal boundary $(X(\infty), T d)$ is a thick spherical building of dimension one. Weyl chambers of $X$ are corresponding to edges of the building $(X(\infty), T d)$ and any edge has length $\pi / 3$. By Theorem 6.1, for any parabolic isometry $f \in \mathrm{SL}(3, \mathbb{R})$ of $X, X_{f}(\infty)$ is one of the following:

(1) an edge,

(2) a closed interval of length $\pi$ consisting of three edges,

(3) the union of an edge $c$ and all edges incident to $c$.

In (3), $X_{f}(\infty)$ has uncountably many edges.

For the irreducible symmetric space $X:=\operatorname{SL}(n, \mathbb{R}) / \mathrm{SO}(n), n \geq 3$, let $f$ be any isometry of $X$. Since for any Weyl chamber $c$ at infinity, $f c \cap c$ is a (possibly empty) face of $c$ and since $\operatorname{rad} c \geq \pi / 6$ (cf. [BH]), we have either $\operatorname{rad} X_{f}(\infty)=0$ or $\geq \pi / 6$.

For any given $\theta \in(0, \pi / 2)$, we have an example with $\operatorname{rad} X_{f}(\infty)=\theta$, where $X$ is a manifold with boundary.

Example 1.6. Let $h$ be a parabolic isometry of $\mathbb{H}^{2}$ and $x$ its fixed point in $\mathbb{H}^{2}(\infty)$. Let $\gamma$ be a ray in $\mathbb{H}^{2}$ tending to $x$, and $b_{\gamma}$ the Busemann function associated with $\gamma$ (see Section 2 for the definition of $b_{\gamma}$ ). Note that $h$ leaves every horosphere $b_{\gamma}^{-1}(t)$ invariant. For an arbitrarily given $\theta \in(0, \pi / 2)$, we consider the closed convex subset

$$
X:=\left\{(p, s) \in \mathbb{H}^{2} \times \mathbb{R}\left|b_{\gamma}(p) \leq-t,\right| s \mid \leq t \sin \theta \text { for some } t \geq 0\right\}
$$

of $\mathbb{H}^{2} \times \mathbb{R} . \quad X$ is a proper CAT $(0)$-space and $(X(\infty), T d)$ is isometric to a closed interval of length $2 \theta$ whose midpoint corresponds to $x$. The product map $\left(h, \mathrm{id}_{\mathbb{R}}\right)$ leaves $X$ invariant, and its restriction, $f$, on $X$ is a parabolic isometry of $X$. Since $X_{f}(\infty)$ coincides with $X(\infty)$, we have $\operatorname{rad} X_{f}(\infty)=\theta$.

1.2. Key ideas of the proof of main theorems. We prove Theorem 1.1 in Section 3 by using the gradient curves for the displacement function, the existence of which is established by Jost and Mayer ([J], [M]). Our proof is based on Schroeder's original argument for Hadamard manifolds in Appendix 3 in [BGS]. Since a CAT(0)-space $X$ is not differentiable in general, we need to investigate the directional derivatives of a Lipschitz continuous, convex function on $X$. It is non-trivial to prove a first variation formula for such a function (see Lemma 3.5).

For Theorem 1.3, the original proof in [BGS] does not seem to work for a CAT(0)space. We take a new approach using the geometry of the Tits ideal boundary $(X(\infty), T d)$ as explained in the following. 
For a topological space $Y, \operatorname{dim}_{C} Y$ is defined as the supremum of the covering dimensions of compact subsets of $Y(\mathrm{cf}$. $[\mathrm{K}])$. A key theorem needed for understanding the set of centers of $X_{f}(\infty)$ is the following.

Theorem 1.7. Let $Y$ be a complete CAT(1)-space of $\operatorname{dim}_{C} Y<\infty$ and diameter $\operatorname{diam} Y \leq \pi / 2$. Then there exists a constant $\delta>0$, which depends only on $\operatorname{dim}_{C} Y$, such that $\operatorname{rad} Y \leq \pi / 2-\delta$. In particular, $C(Y)$ consists of a single point.

Schroeder has shown the same statement if $Y$ is a closed convex subset of the unit sphere of dimension $n$ in Appendix 3 in [BGS]. The basic strategy of the proof is following [BGS], however the proof is more delicate because $Y$ is possibly noncompact. Namely, we cannot avoid a discussion of error estimates (i.e., the estimate of $\delta$ ). We only need $\operatorname{rad} Y<\pi / 2$ for Theorem 1.3.

It is necessary for Theorem 1.7 that $\operatorname{dim}_{C} Y$ is finite. In fact, the inductive limit, $Y$, of the standard spherical $(m-1)$-simplices $\triangle_{1}^{m-1}, m=1,2, \ldots$, given in (1.1) is a complete CAT(1)-space such that $\operatorname{dim}_{C} Y=\infty$, diam $Y=\pi / 2$, and $\operatorname{rad} Y=\pi / 2$.

For applying Theorem 1.7 to $Y:=X_{f}(\infty)$, we need the next result.

Proposition 1.8. For a proper $\mathrm{CAT}(0)$-space $X$ we have

$$
\operatorname{dim}_{C}(X(\infty), T d) \leq \operatorname{dim} X-1,
$$

where $\operatorname{dim} X$ denotes the covering dimension of $X$.

Theorem $\mathrm{C}$ in $[\mathrm{K}]$ implies Proposition 1.8, provided that $X$ has a cocompact group action. For the proof of the proposition, we use a result in [FSY] on the dimension of $X(\infty)$ with sphere topology. There is another way to obtain the proposition using Lemma 11.1 of [L]. We would like to thank A. Lytchak for bringing his work to our attention. We do not know whether $\operatorname{dim}_{C}$ in Proposition 1.8 can be replaced with the covering dimension.

Theorem 1.3 is proved as follows. By Theorem 1.1, $Y:=C\left(X_{f}(\infty)\right)$ has

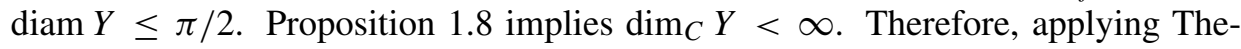
orem 1.7, we obtain Theorem 1.3. The details are given in Section 3.5.

We would like to thank the referee for carefully reading the original manuscript and giving helpful comments.

\section{Preliminaries}

A minimizing geodesic is, by definition, a length-minimizing curve joining two points in a metric space. We assume that all minimizing geodesics have unit speed parameters. Denote by $\gamma_{p q}$ a minimizing geodesic from a point $p$ to a point $q$, and by $[p, q]$ 
its image. A geodesic triangle $\triangle(p, q, r)$ means a triple of minimizing geodesics $\gamma_{p q}, \gamma_{q r}$, and $\gamma_{r p}$ for three points $p, q$, and $r$, called vertices.

For $\kappa \in \mathbb{R}$, let $M_{\kappa}^{2}$ be a complete, simply connected model surface of constant curvature $\kappa$. We set $D_{\kappa}:=\operatorname{diam} M_{\kappa}^{2}$. Note that $D_{\kappa}$ is equal to $\pi / \sqrt{\kappa}$ if $\kappa>0$, and to $\infty$ if $\kappa \leq 0$. We say that a metric space $X$ is a CAT $(\kappa)$-space if the following properties (1) and (2) are satisfied.

(1) Any two points $p, q \in X$ with $d(p, q)<D_{\kappa}$ can be joined by a minimizing geodesic in $X$.

(2) (CAT $(\kappa)$-inequality) Let $\triangle(p, q, r)$ be any geodesic triangle in $X$ with perimeter $<2 D_{\kappa}$ and $\Delta(\tilde{p}, \tilde{q}, \tilde{r})$ a comparison triangle of it in $M_{\kappa}^{2}$, i.e., having the same side lengths as $\Delta(\tilde{p}, \tilde{q}, \tilde{r})$. For any four points $x \in[p, q], y \in[r, p]$, $\tilde{x} \in[\tilde{p}, \widetilde{q}]$, and $\tilde{y} \in[\tilde{r}, \widetilde{p}]$ such that $d(p, x)=d(\tilde{p}, \tilde{x})$ and $d(p, y)=$ $d(\tilde{p}, \tilde{y})$, we have

$$
d(x, y) \leq d(\tilde{x}, \tilde{y}),
$$

where $d$ denotes the distance function.

Let $X$ be a CAT $(\kappa)$-space. A minimizing geodesic $\gamma_{p q}$ joining two points $p, q \in X$ with $d(p, q)<D_{\kappa}$ is unique. For $p \in X$ and $q_{1}, q_{2} \in X \backslash\{p\}$, we denote by $L_{p}\left(\gamma_{p q_{1}}, \gamma_{p q_{2}}\right)$ the angle at $p$ between $\gamma_{p q_{1}}$ and $\gamma_{p q_{2}} . L_{p}$ is a pseudo-distance function on the set of all minimizing geodesics emanating from $p$. The quotient metric space by the relation $L_{p}=0$ is denoted by $\Sigma_{p}^{*} X$. Let $\Sigma_{p} X$ be the $L_{p}$-completion of $\Sigma_{p}^{*} X$, which is called the space of directions at $p$. We denote by $C_{p} X$ the Euclidean cone over $\Sigma_{p} X$, and call this the tangent cone at $p . \Sigma_{p} X$ is a complete CAT(1)-space and $C_{p} X$ is a complete CAT $(0)$-space. We denote by $\dot{\gamma}(0)$ the equivalence class in $\Sigma_{p}^{*} X$ of a minimizing geodesic $\gamma$ from $p$.

Assume that $X$ is a complete CAT( 0$)$-space. Two rays $\gamma, \sigma:[0,+\infty) \rightarrow X$ are said to be asymptotic if $d(\gamma(t), \sigma(t))$ is uniformly bounded for all $t \geq 0$. The ideal boundary $X(\infty)$ of $X$ is defined as the set of all asymptotic equivalence classes of rays in $X . X(\infty)$ is equipped with the sphere topology, which is the restriction of the cone topology (cf. II.8 [BH]) on $X \sqcup X(\infty)$. We denote by $\gamma(\infty)$ the equivalence class in $X(\infty)$ of a ray $\gamma$ in $X$. For any $p \in X$ and $x \in X(\infty)$ there exists a unique ray $\gamma_{p x}:[0, \infty) \rightarrow X$ from $p$ to $\gamma(\infty)=x$.

For $x, y \in X(\infty)$, we set $\angle(x, y):=\sup _{p \in X} L_{p}(x, y)$, the angle distance between $x$ and $y$, where we write $L_{p}(x, y):=L_{p}\left(\gamma_{p x}, \gamma_{p y}\right)$. Note that $\angle$ is a distance function on $X(\infty)$ and is lower semi-continuous with respect to the sphere topology. We remark that if $X$ is proper, then $X(\infty)$ is compact with respect to the sphere topology. The Tits distance on $X(\infty)$, denoted by $T d$, is the interior distance induced from $\angle$. We have $\angle=\min \{T d, \pi\}$. The Tits ideal boundary $(X(\infty), T d)$ of $X$ is a complete CAT(1)-space, which is non-compact in general. The Busemann function $b_{\gamma}: X \rightarrow \mathbb{R}$ associated to a ray $\gamma$ in $X$ is defined as

$$
b_{\gamma}(p):=\lim _{t \rightarrow \infty}\{d(p, \gamma(t))-t\} .
$$


This is a 1-Lipschitz continuous, convex function with $b_{\gamma}(\gamma(0))=0$.

A subset $A$ of a metric space $X$ is said to be convex in $X$ if any $x, y \in A$ can be joined by a minimizing geodesic and the image of every such geodesic is contained in $A$. If this condition holds only for any $x, y \in A$ with $d(x, y)<r$, then $A$ is said to be $r$-convex in $X$.

Let $B$ be a closed subset of a metric space $X$. We define a function $d_{B}: X \rightarrow$ $[0, \infty)$ by $d_{B}(p):=d(p, B)$, the distance function from $B$. For $p \in X \backslash B$, we denote by $\gamma_{p B}$ a minimizing geodesic in $X$ from $p$ to $B$, i.e., to a point $q \in B$ with $d_{B}(p)=d(p, q)$.

Assume that $B$ is a closed, convex subset of a complete CAT(0)-space. Then, for any $p \in X$ there exists a unique point $q \in B$ with $d_{B}(p)=d_{B}(p, q)$, in particular, $\gamma_{p q}=\gamma_{p B}$. We note that $d_{B}$ is a 1-Lipschitz continuous, convex function.

\section{Estimate of radii of fixed point sets}

We prove Theorem 1.1.

3.1. Directional derivatives of convex functions. Let $X$ be a complete CAT(0)space and $F: X \rightarrow \mathbb{R}$ a locally Lipschitz continuous, convex function. We discuss the directional derivatives of $F$. For any geodesic $\gamma$ in $X, F \circ \gamma$ has the left and right derivatives. Recall that the tangent cone $C_{p} X$ is the quotient space $[0,+\infty) \times$ $\Sigma_{p} X /\{0\} \times \Sigma_{p} X$. We identify the subspace $\{1\} \times \Sigma_{p} X$ of $C_{p} X$ with $\Sigma_{p} X$. Denote any element $(t, v) \in C_{p} X$ by $t v$ and define $|t v|:=t$. Let $C_{p}^{*} X:=[0, \infty) \times \Sigma_{p}^{*} X /\{0\} \times$ $\Sigma_{p}^{*} X \subset C_{p} X$. The directional derivative $D_{p} F: C_{p}^{*} X \rightarrow \mathbb{R}$ of $F$ at a point $p \in X$ is defined as

$$
D_{p} F(t v):=\lim _{s \rightarrow 0+} \frac{F\left(\gamma_{v}(s)\right)-F\left(\gamma_{v}(0)\right)}{s} t,
$$

where $\gamma_{v}$ is a minimizing geodesic from $p$ with $v=\dot{\gamma}_{v}(0)$. The existence of the limit above is guaranteed by the convexity of $F . D_{p} F(t v)$ is independent of the choice of $\gamma_{v} . D_{p} F$ extends to a unique Lipschitz continuous function on $C_{p} X$, which is convex (cf. Lemma 2.4 in $[\mathrm{K}]$ ). Moreover, it is linear along each ray from the vertex $o_{p}$ of $C_{p} X$.

Assume that $\Sigma_{p} X$ is compact for every $p \in X$. We say that a point $p \in X$ is a critical point of $F$ if $D_{p} F(u) \geq 0$ for every $u \in \Sigma_{p} X$. Note that, by the convexity of $F$, a point is critical for $F$ if and only if it is a minimizer of $F$. For more general functions, such as $c$-convex functions (cf. [BBI]), this is not true and we still have some local properties stated below, e.g. Theorem 3.1 and Lemma 3.5. By the convexity of $D_{p} F$ and the compactness of $\Sigma_{p} X$, for any non-critical point $p$ of $F$, there exists a unique direction $u_{p} \in \Sigma_{p} X$ where $\left.D_{p} F\right|_{\Sigma_{p} X}$ attains its minimum $(<0)$. We call $u_{p}$ the gradient direction of $-F$ at $p$. Define the gradient vector $\operatorname{grad}_{p}(-F) \in C_{p} X$ 
of $-F$ at a point $p$ by

$$
\operatorname{grad}_{p}(-F):=\left|D_{p} F\left(u_{p}\right)\right| u_{p} \in C_{p} X
$$

if $p$ is non-critical, and by $\operatorname{grad}_{p}(-F):=o_{p}$ (the vertex) if $p$ is critical. It follows that $\left|\operatorname{grad}_{p}(-F)\right|=-D_{p} F\left(u_{p}\right)$.

3.2. Jost-Mayer's gradient curves. The following theorem is a restricted version of a result in $[\mathrm{J}],[\mathrm{M}]$.

Theorem 3.1 ([J], [M]). Let $X$ be a complete $\mathrm{CAT}(0)$-space such that $\Sigma_{p} X$ is compact for every $p \in X$, and let $F: X \rightarrow \mathbb{R}$ be a convex function. Then, for every $p \in X$ there exists a Lipschitz continuous curve $c_{p}:[0, \infty) \rightarrow X$ from $p=c_{p}(0)$, called the gradient curve from $p$ for $-F$, such that for any $t \geq 0$ we have

$$
\begin{aligned}
\lim _{s \rightarrow 0+} \frac{d\left(c_{p}(t+s), c_{p}(t)\right)}{s} & =\lim _{s \rightarrow 0+} \frac{-F \circ c_{p}(t+s)+F \circ c_{p}(t)}{d\left(c_{p}(t+s), c_{p}(t)\right)} \\
& =\limsup _{q \rightarrow c_{p}(t)} \frac{-F(q)+F\left(c_{p}(t)\right)}{d\left(q, c_{p}(t)\right)} \\
& =\left|\operatorname{grad}_{c_{p}(t)}(-F)\right|,
\end{aligned}
$$

$$
\left(F \circ c_{p}\right)_{+}^{\prime}(t)=\left|\operatorname{grad}_{c_{p}(t)}(-F)\right|^{2},
$$

where $\left(F \circ c_{p}\right)_{+}^{\prime}(t)$ is the right derivative of $F \circ c_{p}$ at $t$. Moreover, for any $r \geq 0$, the gradient curve $c_{c_{p}(t)}$ from $c_{p}(t)$ for $-F$ satisfies

$$
c_{c_{p}(t)}(r)=c_{p}(t+r) .
$$

Under the same assumption as in Theorem 3.1, we have:

Lemma 3.2. For the gradient curve $c_{p}$ from $p$ of $-F$, the right tangent vector $\left(\dot{c}_{p}\right)_{+}(0) \in C_{p} X$ exists and coincides with $\operatorname{grad}_{p}(-F)$.

Proof. By taking a sequence $\left\{s_{i}\right\}$ with $s_{i} \rightarrow 0+$, we have a limit $v \in \Sigma_{p} X$ of the direction $\dot{\gamma}_{p c_{p}\left(s_{i}\right)}(0)$ as $i \rightarrow \infty$. By Theorem 3.1(1), $D_{p} F(v)$ must be equal to $D_{p} F\left(u_{p}\right)=-\left|\operatorname{grad}_{p}(-F)\right|$. We see that $v=u_{p}$ by the uniqueness of the gradient direction $u_{p}$.

3.3. First variation formula. The following is well known.

Lemma 3.3. Let $X$ be a complete $\mathrm{CAT}(0)$-space. 
Vol. 81 (2006)

(1) Let $B$ be a closed, convex subset of $X$. Then for any $p \in X \backslash B$ and $v \in \Sigma_{p} X$ we have

$$
D_{p} d_{B}(v)=-\cos L_{p}\left(\dot{\gamma}_{p B}(0), v\right) .
$$

(2) Let $\gamma$ be a ray in $X$. Then for any $p \in X$ and $v \in \Sigma_{p} X$ we have

$$
D_{p} b_{\gamma}(v)=-\cos L_{p}\left(\dot{\gamma}_{p \gamma(\infty)}(0), v\right) .
$$

Proof. (1) follows from a standard argument (cf. Section 4.5 of [BBI]).

We prove (2). Set $B_{t}:=b_{\gamma}^{-1}(-\infty,-t]$ for $t>0 . B_{t}$ is convex in $X$. Let $p \in X$ be any point. If $t>0$ is large enough for $p$, then $p \in X \backslash B_{t}$ and $d_{B_{t}}(p)=b_{\gamma}(p)+t$ (cf. Proposition II.8.22 in [BH]), which and (1) imply (2).

Some variants of Lemma 3.3(1) are seen in Section 4.5 of [BBI]. Note that the CAT(0)-condition for $X$ is not essential for Lemma 3.3.

To prove a first variation formula for convex functions, we need a lemma.

Lemma 3.4. Let $S$ be a sector in $\mathbb{E}^{2}$ bounded by two distinct rays from the origin o. Let $F: S \rightarrow \mathbb{R}$ be a function that is linear along each ray from $o$. If the directional derivative $D_{u} F: C_{u} S \rightarrow \mathbb{R}$ of $F$ at a point $u \in S \backslash\{o\}$ exists, then $D_{u} F$ is linear on $C_{u} S$.

Lemma 3.4 is shown by a standard argument. We omit the proof.

We prove the following first variation formula.

Lemma 3.5. Let $F: X \rightarrow \mathbb{R}$ be a locally Lipschitz continuous, convex function on a complete CAT(0)-space $X$. Let $p \in X$ be a non-critical point of $F$ such that $\Sigma_{p} X$ is compact. Then for any $v \in \Sigma_{p} X$ we have

$$
D_{p} F(v) \geq-\left|\operatorname{grad}_{p}(-F)\right| \cos L_{p}\left(u_{p}, v\right),
$$

where $u_{p} \in \Sigma_{p} X$ is the gradient direction of $-F$ at $p$.

Proof. Let $v \in \Sigma_{p} X$ be a direction. If $L_{p}\left(u_{p}, v\right)=0$, the lemma is obvious. In the case where $L_{p}\left(u_{p}, v\right)=\pi$, the minimizing geodesic $\gamma_{u_{p} v}$ in $C_{p} X$ from $u_{p}$ to $v$ passes through the vertex $o_{p}$, so that the convexity of $D_{p} F$ along $\gamma_{u_{p} v}$ implies the lemma.

We assume that $0<L_{p}\left(u_{p}, v\right)<\pi$. Now consider the second derivative $D_{u_{p}} D_{p} F: C_{u_{p}} C_{p} X \rightarrow \mathbb{R}$. Let $S \subset C_{p} X$ be the 2-dimensional flat sector generated by $\gamma_{u_{p} v} . S$ is convex in $C_{p} X$. We set $\xi:=\dot{\gamma}_{u_{p} v}(0)$ and $\eta:=\dot{\gamma}_{u_{p} o_{p}}(0)$, both which belong to $\Sigma_{u_{p}} S$. Note that $C_{u_{p}} S$ is a flat half plane in $C_{u_{p}} C_{p} X$. Take the direction $\zeta \in \Sigma_{u_{p}} S$ perpendicular to $\eta$. Setting $\theta:=L_{u_{p}}\left(o_{p}, v\right)$, we see 
$\xi=(\cos \theta) \eta+(\sin \theta) \zeta$. Since Lemma 3.4 implies the linearity of $D_{u_{p}} D_{p} F$, we have

$$
D_{u_{p}} D_{p} F(\xi)=D_{u_{p}} D_{p} F(\eta) \cos \theta+D_{u_{p}} D_{p} F(\zeta) \sin \theta .
$$

The linearity of $D_{p} F$ along $\gamma_{u_{p} o_{p}}$ shows that $D_{u_{p}} D_{p} F(\eta)=-D_{p} F\left(u_{p}\right)>0$. Since $u_{p}$ is the minimum point of $D_{p} F$ on $\Sigma_{p} X$, we have $D_{u_{p}} D_{p} F(\zeta) \geq 0$. Thus, by noting $0<\theta<\pi / 2$,

$$
D_{u_{p}} D_{p} F(\xi) \geq-D_{p} F\left(u_{p}\right) \cos \theta(>0) .
$$

It follows that the distance between $u_{p}$ and $v$ in $C_{p} X$ is equal to $2 \cos \theta$, so that, by the convexity of $D_{p} F$ along $\gamma_{u_{p} v}$,

$$
D_{p} F(v) \geq D_{p} F\left(u_{p}\right)+2 D_{u_{p}} D_{p} F(\xi) \cos \theta .
$$

Combining (3.1) and (3.2) yields

$$
D_{p} F(v) \geq-D_{p} F\left(u_{p}\right) \cos 2 \theta=D_{p} F\left(u_{p}\right) \cos L_{p}\left(u_{p}, v\right),
$$

which completes the proof of Lemma 3.5.

Note that the equality in Lemma 3.5 does not necessarily hold. Lemma 3.5 remains true for a locally Lipschitz continuous, $c$-convex function $F$ on a locally $\mathrm{CAT}(\kappa)$-space $X, c, \kappa \in \mathbb{R}$.

3.4. Monotone points. Let $X$ be a complete CAT( 0 )-space and $F: X \rightarrow \mathbb{R}$ a convex function. The following terminology was introduced by Eberlein in Section 4.1 of [E] for a Riemannian manifold. A point $x \in X(\infty)$ is said to be $F$-monotone if there exists a ray $\gamma:[0, \infty) \rightarrow X$ with $x=\gamma(\infty)$ such that $F \circ \gamma(t)$ is monotone non-increasing in $t \geq 0$. We denote by $X_{F}(\infty)$ the set of all $F$-monotone points in $X(\infty)$, called the $F$-monotone set. For an isometry $f$ of $X$, we recall the displacement function $d_{f}(p):=d(p, f(p))$, which is a 1-Lipschitz continuous, convex function on $X$. For a ray $\gamma$ in $X, \gamma(\infty)$ is $d_{f}$-monotone if and only if $f \circ \gamma$ is asymptotic to $\gamma$. This leads to $X_{d_{f}}(\infty)=X_{f}(\infty)$.

The following lemma is obtained by the same discussion as in Section 4.1 of [E]. We omit the proof.

Lemma 3.6. Let $F: X \rightarrow \mathbb{R}$ be a convex function.

(1) For a point $x \in X(\infty)$, the following properties are equivalent to each other.

(a) $x$ is F-monotone.

(b) For any ray $\gamma$ with $x=\gamma(\infty), F \circ \gamma(t)$ is monotone non-increasing in $t \geq 0$. 
(c) There exists a sequence $\left\{p_{i}\right\}$ of points in $X$ converging to $x$ in the cone topology such that $F\left(p_{i}\right)$ is uniformly bounded from above.

(2) $X_{F}(\infty)$ is closed with respect to the sphere topology.

(3) If $X$ is proper, then $X_{F}(\infty)$ is a closed, $\pi$-convex subset of $(X(\infty), T d)$.

3.5. Proof of Theorem 1.1. We prove the theorem in the same way as in [E] by using Lemma 3.5. Let $X$ be a proper CAT(0)-space such that $\Sigma_{p} X$ is compact for every $p \in X$, and $f$ a parabolic isometry of $X$. Since the displacement function $d_{f}$ has no minimal (or critical) point in $X$, we have the gradient direction $u_{p}$ of $-d_{f}$ at any $p \in X$, which satisfies $D_{p} d_{f}\left(u_{p}\right)<0$. We fix a point $p \in X$ and take the gradient curve $c_{p}$ from $p$ for $-d_{f}$. By Lemma 3.2, the right tangent vector $\left(\dot{c}_{p}\right)_{+}(t) \in C_{p} X$ satisfies $\left(\dot{c}_{p}\right)_{+}(t)=\operatorname{grad}_{c_{p}(t)}\left(-d_{f}\right)$ for any $t \geq 0$. It follows from Theorem 3.1(1) that $d_{f} \circ c_{p}(t)$ is strictly monotone decreasing in $t \geq 0$. There exists a sequence $t_{i} \rightarrow \infty$ such that $c_{p}\left(t_{i}\right)$ converges to some point $x \in X(\infty)$ in the cone topology. Lemma 3.6(1) implies $x \in X_{f}(\infty)$.

We take any $y \in X_{f}(\infty)$ and fix it. It suffices to prove that $T d(x, y) \leq \pi / 2$. Let $v_{t}:=\dot{\gamma}_{c_{p}(t) y}(0)$. Consider the Busemann function $b:=b_{\gamma_{p y}}$ associated with $\gamma_{p y}$. Since $y$ is $d_{f}$-monotone and by Theorem 3.1(1), Lemma 3.3(2), and Lemma 3.5, we have

$$
\left(b \circ c_{p}\right)_{+}^{\prime}(t)=-\left|\operatorname{grad}_{c_{p}(t)}\left(-d_{f}\right)\right| \cos L_{c_{p}(t)}\left(u_{c_{p}(t)}, v_{t}\right) \leq D_{c_{p}(t)} d_{f}\left(v_{t}\right) \leq 0
$$

for any $t \geq 0$, and hence $b \circ c_{p}(t)$ is monotone non-increasing in $t$. By Lemma 3.6(1), $x$ is $b$-monotone and, for any $q \in X, b \circ \gamma_{q x}(t)$ is monotone non-increasing in $t$. It follows from Lemma 3.3(2) that

$$
-\cos L_{q}(x, y)=\left(b \circ \gamma_{q x}\right)_{+}^{\prime}(0) \leq 0,
$$

which proves $T d(x, y) \leq \pi / 2$.

Since $(X(\infty), T d)$ is $\operatorname{CAT}(1), X_{f}(\infty)$ is contractible. This completes the proof of Theorem 1.1.

Let $X$ be as in Theorem 1.1. Then we have $\operatorname{rad} X_{F}(\infty) \leq \pi / 2$ for any locally Lipschitz continuous, convex function $F$ on $X$ with no minimum in $X$.

\section{Dimension of Tits ideal boundaries}

We need the following to prove Proposition 1.8.

Proposition 4.1 ([FSY]). Let $X$ be a proper CAT(0)-space. Then, the covering dimension of $X(\infty)$ for the sphere topology satisfies

$$
\operatorname{dim} X(\infty) \leq \operatorname{dim} X-1 .
$$


Proof of Proposition 1.8. By Proposition 4.1, it suffices to show that

$$
\operatorname{dim}_{C}(X(\infty), T d) \leq \operatorname{dim} X(\infty) .
$$

We consider the identity map $\iota:(X(\infty), T d) \rightarrow X(\infty)$, which is continuous. Take any compact subset $K \subset(X(\infty), T d)$. Since $X(\infty)$ is Hausdorff, $\left.\iota\right|_{K}: K \rightarrow \iota(K)$ is a homeomorphism. Thus, we have $\operatorname{dim} K=\operatorname{dim} \iota(K) \leq \operatorname{dim} X(\infty)$. This completes the proof.

We denote by $\triangle^{n}=\Delta^{n}\left(a_{0}, a_{1}, \ldots, a_{n}\right)$ a (closed) $n$-simplex with vertices $a_{0}, a_{1}, \ldots, a_{n}$. Let $F_{i} \subset \partial \triangle^{n}$ be the $(n-1)$-simplex that is the opposite face to $a_{i}$, where $\partial \triangle^{n}$ is the boundary of $\triangle^{n}$. We say that a map $\psi$ from $\triangle^{n}$ to a set collapses $\partial \triangle^{n}$ if

$$
\psi\left(F_{0}\right) \cap \psi\left(F_{1}\right) \cap \cdots \cap \psi\left(F_{n}\right) \neq \emptyset .
$$

The following is a consequence of Sperner's lemma (cf. 2.1 in $[\mathrm{F}]$ ).

Lemma 4.2. Let $Y$ be a Hausdorff space of $\operatorname{dim} Y \leq n-1, n \geq 1$. Then any continuous map $\psi: \Delta^{n} \rightarrow Y$ collapses $\partial \triangle^{n}$.

Proof. Suppose that there exists a continuous map $\psi: \triangle^{n} \rightarrow Y$ that does not collapse $\partial \triangle^{n}$. We set $U_{i}:=Y \backslash \psi\left(F_{i}\right), i=0,1, \ldots, n$, which are open in the Hausdorff space $Y$. Since $\psi$ does not collapse $\partial \triangle^{n},\left\{U_{i}\right\}_{i=0}^{n}$ is an open covering of $Y$. By $\operatorname{dim} Y \leq n-1$, there exists a refinement $\left\{V_{i}\right\}$ of $\left\{U_{i}\right\}$ of order at most $n$. Since $\psi$ is continuous and the order of $\left\{V_{i}\right\}$ is at most $n$, we can take a sufficiently refined triangulation of $\Delta^{n}$ such that for each simplex $s$ of it, $\psi(s)$ intersects at most $n$ members of $\left\{V_{i}\right\}$. Then we give a label by $i=0,1, \ldots, n$ to each vertex of the refinement as follows. A label of a vertex $a$ is $i$ if $\psi(a) \in V_{i}$, which implies that this label is a Sperner label on $\Delta^{n}$. Namely, each original vertex $a_{i}$ has the label $i$, and each vertex in the refinement contained in a $j$-dimensional simplex $\triangle^{j}=\triangle^{j}\left(a_{i_{0}}, a_{i_{1}}, \ldots, a_{i_{j}}\right)$ is labelled by one of $i_{0}, i_{1}, \ldots, i_{j}$; e.g., a vertex contained in $F_{i}$ does not have the label $i$. Therefore, by Sperner's lemma there exists at least one $n$-simplex $s^{n}$ in the refined triangulation of $\Delta^{n}$ such that the vertices of $s^{n}$ have the $n+1$ different labels, $0,1, \ldots, n$.

On the other hand, since $\psi\left(s^{n}\right)$ is contained in at most $n$ different $V_{i}$ 's, the simplex $s^{n}$ has at most $n$ different labels. This is a contradiction.

Lemma 4.2 plays a key role in the proof of Theorem 1.7 in Section 5. As another application of Lemma 4.2, we have:

Proposition 4.3. Let $Y$ be a CAT(1)-space of $\operatorname{dim}_{C} Y \leq m, m \geq 1$. Then, for any embedding $\psi$ from an $m$-sphere $\mathbb{S}^{m}$ into $Y$ we have $\operatorname{rad} \psi\left(\mathbb{S}^{m}\right) \geq \pi$. In particular, if $m=1$, then $Y$ is locally an $\mathbb{R}$-tree. 
Proof. Suppose that there exists an embedding $\psi: \mathbb{S}^{m} \rightarrow Y$ satisfying $\operatorname{rad} \psi\left(\mathbb{S}^{m}\right)<$ $\pi$. Since $Y$ is $\operatorname{CAT}(1), \psi\left(\mathbb{S}^{m}\right)$ is contractible in $Y$. Hence, for a closed $(m+1)$ disk $D^{m+1}$ there is a continuous extension $\bar{\psi}: D^{m+1} \rightarrow Y$ of $\psi$. By identifying $D^{m+1}$ with an $(m+1)$-simplex, $\bar{\psi}$ does not collapse $\partial D^{m+1}$ and $\operatorname{dim} \bar{\psi}\left(D^{m+1}\right) \leq$ $\operatorname{dim}_{C} Y \leq m$. This contradicts Lemma 4.2.

Remark 4.4. Let $X$ be a proper CAT(0)-space of $\operatorname{dim} X \leq n$. By Proposition 1.8, we can apply Proposition 4.3 to $Y=(X(\infty), T d)$ and $m=n-1$.

\section{CAT(1)-spaces of small diameter}

In this section we shall prove Theorems 1.3 and 1.7.

5.1. Small triangles. Let $Y$ be a CAT(1)-space. For $x, y, z \in Y$ we set $L_{x}(y, z):=$ $L_{x}\left(\gamma_{x y}, \gamma_{x z}\right)$. Denote the image of $\gamma_{x y}$ by $[x, y]$. Let $\Delta=\triangle\left(a_{0}, a_{1}, a_{2}\right)$ be a geodesic triangle in $Y$ with sides $\left[a_{0}, a_{1}\right],\left[a_{1}, a_{2}\right],\left[a_{2}, a_{0}\right]$, and $\widetilde{\Delta}=\Delta\left(\widetilde{a}_{0}, \widetilde{a}_{1}, \widetilde{a}_{2}\right)$ a comparison triangle in $\mathbb{S}^{2}(1)$ of $\Delta$ with the same side-lengths as $\Delta$. Recall that $L_{a_{i}}\left(a_{j}, a_{k}\right) \leq L_{a_{i}}\left(\widetilde{a}_{j}, \tilde{a}_{k}\right)$ for distinct $i, j, k=0,1,2$. We say that $\triangle\left(a_{0}, a_{1}, a_{2}\right)$ is small if $d\left(a_{i}, a_{j}\right) \leq \pi / 2$ for any $i, j=0,1,2$. If $\triangle\left(a_{0}, a_{1}, a_{2}\right)$ is small, then we have $d\left(a_{2}, x\right) \leq \pi / 2$ for any $x \in\left[a_{0}, a_{1}\right]$ by the CAT(1)-inequality. If $\Delta\left(a_{0}, a_{1}, a_{2}\right)$ is small and if $d\left(a_{2}, x\right)=\pi / 2$ for some $x \in\left[a_{0}, a_{1}\right] \backslash\left\{a_{0}, a_{1}\right\}$, then the triangle is an isosceles triangle and bounds a convex spherical surface.

As usual, $O(\varepsilon)$ denotes Landau's symbol, i.e., some universal function such that $\lim \sup _{\varepsilon \rightarrow 0}|O(\varepsilon)| / \varepsilon$ is finite. We assume that $O(\varepsilon)$ is positive.

For the proof of Theorem 1.7, we first show:

Lemma 5.1. Let $\varepsilon \in(0,1)$ be a positive number. Let $\Delta=\triangle\left(a_{0}, a_{1}, a_{2}\right)$ and $\triangle^{\prime}=\triangle\left(a_{0}^{\prime}, a_{1}^{\prime}, a_{2}^{\prime}\right)$ be small geodesic triangles in $Y$ and in $\mathbb{S}^{2}(1)$, respectively. Then the following holds:

(1) if $\left|d\left(a_{i}, a_{j}\right)-d\left(a_{i}^{\prime}, a_{j}^{\prime}\right)\right| \leq \varepsilon$ for any $i, j=0,1,2$ and if $d\left(a_{0}, a_{j}\right) \geq \varepsilon^{1 / 2}$ for each $j=1,2$, then we have

$$
\left\llcorner_{a_{0}}\left(a_{1}, a_{2}\right)<\left\llcorner_{a_{0}^{\prime}}\left(a_{1}^{\prime}, a_{2}^{\prime}\right)+O\left(\varepsilon^{1 / 2}\right) ;\right.\right.
$$

(2) if $L_{a_{0}}\left(a_{1}, a_{2}\right) \geq L_{a_{0}^{\prime}}\left(a_{1}^{\prime}, a_{2}^{\prime}\right)-\varepsilon$ and $\left|d\left(a_{0}, a_{j}\right)-d\left(a_{0}^{\prime}, a_{j}^{\prime}\right)\right| \leq \varepsilon$ for each $j=1,2$, then we have

$$
d\left(a_{1}, a_{2}\right)>d\left(a_{1}^{\prime}, a_{2}^{\prime}\right)-O(\varepsilon) .
$$

Proof. (1) Let $\widetilde{\triangle}=\Delta\left(\widetilde{a}_{0}, \tilde{a}_{1}, \tilde{a}_{2}\right)$ be a comparison triangle in $\mathbb{S}^{2}(1)$ of $\triangle$. Since $Y$ is CAT(1), we have $L_{a_{0}}\left(a_{1}, a_{2}\right) \leq L_{a_{0}}\left(\widetilde{a}_{1}, \widetilde{a}_{2}\right)$. By the assumption of $\triangle$ and $\triangle^{\prime}$, we have the conclusion of (1). 
We omit the proof of (2).

We next prove the following lemma.

Lemma 5.2. Let $\varepsilon \in(0,1)$, and let $\triangle=\triangle\left(a_{0}, a_{1}, a_{2}\right)$ be a small geodesic triangle in $Y$. Assume that there exists a point $y \in\left[a_{0}, a_{1}\right]$ such that $\min _{i=0,1} d\left(a_{i}, y\right) \geq \varepsilon^{1 / 2}$ and $d\left(a_{2}, y\right) \geq \pi / 2-\varepsilon$. Then we have

(1) $\mid\left\llcorner_{y}\left(a_{2}, a_{i}\right)-\pi / 2 \mid<O\left(\varepsilon^{1 / 2}\right), i=0,1\right.$,

(2) $d\left(a_{2}, x\right)>\pi / 2-O\left(\varepsilon^{1 / 2}\right)$

for any $x \in\left[a_{0}, a_{1}\right]$.

Proof. (1) Let $\triangle_{i}^{\prime}=\triangle\left(y^{\prime}, a_{i}^{\prime}, a_{2}^{\prime}\right), i=0,1$, be two spherical triangles in $\mathbb{S}^{2}(1)$ such that $d\left(y^{\prime}, a_{i}^{\prime}\right)=d\left(y, a_{i}\right), d\left(a_{i}^{\prime}, a_{2}^{\prime}\right)=d\left(a_{i}, a_{2}\right)$, and $d\left(a_{2}^{\prime}, y^{\prime}\right)=\pi / 2$. Since each $\triangle_{i}^{\prime}$ is small, we have $L_{y^{\prime}}\left(a_{2}^{\prime}, a_{i}^{\prime}\right) \leq \pi / 2$. By $d\left(a_{2}, y\right) \geq \pi / 2-\varepsilon$, we have $\left|d\left(a_{2}, y\right)-d\left(a_{2}^{\prime}, y^{\prime}\right)\right| \leq \varepsilon$. Applying Lemma 5.1(1) to $\triangle\left(y, a_{i}, a_{2}\right)$ and $\triangle_{i}^{\prime}$ yields that $L_{y}\left(a_{2}, a_{i}\right)<\pi / 2+O\left(\varepsilon^{1 / 2}\right)$. Therefore, by $\pi \leq L_{y}\left(a_{2}, a_{0}\right)+L_{y}\left(a_{2}, a_{1}\right)$ we have $L_{y}\left(a_{2}, a_{i}\right)>\pi / 2-O\left(\varepsilon^{1 / 2}\right)$.

(2) For any given $x \in\left[a_{0}, a_{1}\right] \backslash\{y\}$, let us take a small spherical isosceles triangle $\Delta^{\prime \prime}=\triangle\left(y^{\prime \prime}, x^{\prime \prime}, a_{2}^{\prime \prime}\right)$ such that $d\left(a_{2}^{\prime \prime}, x^{\prime \prime}\right)=d\left(a_{2}^{\prime \prime}, y^{\prime \prime}\right)=\pi / 2$ and $d\left(x^{\prime \prime}, y^{\prime \prime}\right)=$ $d(x, y)$. Since $L_{y^{\prime \prime}}\left(a_{2}^{\prime \prime}, x^{\prime \prime}\right)=\pi / 2$ and by (1) we have $L_{y}\left(a_{2}, x\right)>L_{y^{\prime \prime}}\left(a_{2}^{\prime \prime}, x^{\prime \prime}\right)-$ $O\left(\varepsilon^{1 / 2}\right)$. Applying Lemma 5.1(2) to $\triangle\left(y, x, a_{2}\right)$ and $\triangle^{\prime \prime}$ shows (2).

Lemma 5.3. Let $\varepsilon \in(0,1)$ be a positive number. For two small geodesic triangles $\triangle=\triangle\left(a_{0}, a_{1}, a_{2}\right)$ in $Y$ and $\triangle^{\prime}=\triangle\left(a_{0}^{\prime}, a_{1}^{\prime}, a_{2}^{\prime}\right)$ in $\mathbb{S}^{2}(1)$, we assume that

(1) $d\left(a_{2}, x\right)>\pi / 2-\varepsilon$ for any $x \in\left[a_{0}, a_{1}\right]$;

(2) $d\left(a_{2}^{\prime}, x^{\prime}\right)=\pi / 2$ for any $x^{\prime} \in\left[a_{0}^{\prime}, a_{1}^{\prime}\right]$;

(3) $\left|d\left(a_{0}, a_{1}\right)-d\left(a_{0}^{\prime}, a_{1}^{\prime}\right)\right|<\varepsilon$.

For any four points $x_{i} \in\left[a_{2}, a_{i}\right], x_{i}^{\prime} \in\left[a_{2}^{\prime}, a_{i}^{\prime}\right], i=0,1$, such that

$$
d\left(a_{2}, x_{i}\right) / d\left(a_{2}, a_{i}\right)=d\left(a_{2}^{\prime}, x_{i}^{\prime}\right) / d\left(a_{2}^{\prime}, a_{i}^{\prime}\right),
$$

we have

$$
\left|d\left(x_{0}, x_{1}\right)-d\left(x_{0}^{\prime}, x_{1}^{\prime}\right)\right|<O\left(\varepsilon^{1 / 4}\right) .
$$

Proof. Take such four points $x_{0}, x_{1}, x_{0}^{\prime}$, and $x_{1}^{\prime}$. We may assume that $d\left(a_{0}^{\prime}, a_{1}^{\prime}\right) \geq$ $4 \varepsilon^{1 / 2}$. Note that $d\left(a_{0}, a_{1}\right) \geq 3 \varepsilon^{1 / 2}$. Take $\bar{y}_{i} \in\left[a_{0}, a_{1}\right]$ and $\bar{y}_{i}^{\prime} \in\left[a_{0}^{\prime}, a_{1}^{\prime}\right]$ with $d\left(a_{i}, \bar{y}_{i}\right)=d\left(a_{i}^{\prime}, \bar{y}_{i}^{\prime}\right)=\varepsilon^{1 / 2}$ for $i=0,1$. Let $y_{i} \in\left[a_{2}, \bar{y}_{i}\right]$ and $y_{i}^{\prime} \in\left[a_{2}^{\prime}, \bar{y}_{i}^{\prime}\right]$ be the points determined by

$$
\frac{d\left(a_{2}, y_{i}\right)}{d\left(a_{2}, \bar{y}_{i}\right)}=\frac{d\left(a_{2}, x_{i}\right)}{d\left(a_{2}, a_{i}\right)}, \quad \frac{d\left(a_{2}^{\prime}, y_{i}^{\prime}\right)}{d\left(a_{2}^{\prime}, \bar{y}_{i}^{\prime}\right)}=\frac{d\left(a_{2}^{\prime}, x_{i}^{\prime}\right)}{d\left(a_{2}^{\prime}, a_{i}^{\prime}\right)}
$$


(cf. Figure 1). Let $\widetilde{\Delta}=\Delta\left(\widetilde{\bar{y}}_{0}, \widetilde{\bar{y}}_{1}, \widetilde{a}_{2}\right)$ be a spherical comparison triangle in $\mathbb{S}^{2}(1)$ of $\triangle=\triangle\left(\bar{y}_{0}, \bar{y}_{1}, a_{2}\right)$, and $\widetilde{y}_{0}, \widetilde{y}_{1} \in \widetilde{\triangle}$ the corresponding points to $y_{0}, y_{1}$. Considering the two geodesic triangles $\widetilde{\Delta}$ and $\Delta\left(\bar{y}_{0}^{\prime}, \bar{y}_{1}^{\prime}, a_{2}^{\prime}\right)$ in $\mathbb{S}^{2}(1)$, we have $\mid d\left(\tilde{y}_{0}, \widetilde{\bar{y}}_{1}\right)-$ $d\left(y_{0}^{\prime}, \bar{y}_{1}^{\prime}\right)|| d,\left(\tilde{y}_{0}, \tilde{y}_{1}\right)-d\left(y_{0}^{\prime}, y_{1}^{\prime}\right) \mid<O(\varepsilon)$,

$$
d\left(y_{0}, \bar{y}_{1}\right)<d\left(y_{0}^{\prime}, \bar{y}_{1}^{\prime}\right)+O(\varepsilon),
$$

and $d\left(y_{0}, y_{1}\right)<d\left(y_{0}^{\prime}, y_{1}^{\prime}\right)+O(\varepsilon)$. By $d\left(x_{i}, y_{i}\right), d\left(x_{i}^{\prime}, y_{i}^{\prime}\right)<O\left(\varepsilon^{1 / 2}\right)$, we have $d\left(x_{0}, x_{1}\right)<d\left(x_{0}^{\prime}, x_{1}^{\prime}\right)+O\left(\varepsilon^{1 / 2}\right)$. To obtain the opposite inequality, it suffices to prove

$$
d\left(y_{0}, y_{1}\right)>d\left(y_{0}^{\prime}, y_{1}^{\prime}\right)-O\left(\varepsilon^{1 / 4}\right) .
$$
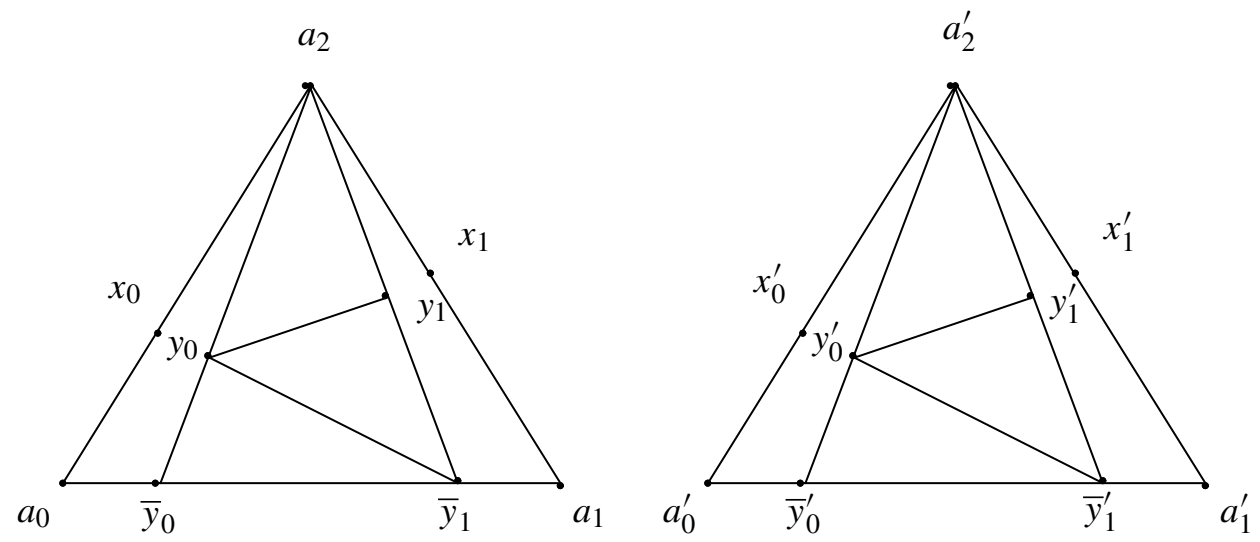

Figure 1. $\Delta=\triangle\left(a_{0}, a_{1}, a_{2}\right)$ and $\Delta^{\prime}=\triangle\left(a_{0}^{\prime}, a_{1}^{\prime}, a_{2}^{\prime}\right)$.

Applying Lemma 5.2(1) to $\triangle\left(a_{0}, \bar{y}_{1}, a_{2}\right)$ and $\triangle\left(\bar{y}_{0}, a_{1}, a_{2}\right)$ yields

$$
\pi / 2-O\left(\varepsilon^{1 / 2}\right)<L_{\bar{y}_{0}}\left(a_{2}, \bar{y}_{1}\right), L_{\bar{y}_{1}}\left(a_{2}, \bar{y}_{0}\right)<\pi / 2+O\left(\varepsilon^{1 / 2}\right) .
$$

Consider $\Delta\left(\bar{y}_{0}, \bar{y}_{1}, y_{0}\right)$ and $\Delta\left(\bar{y}_{0}^{\prime}, \bar{y}_{1}^{\prime}, y_{0}^{\prime}\right)$. By $L_{\bar{y}_{0}^{\prime}}\left(a_{2}^{\prime}, \bar{y}_{1}^{\prime}\right)=\pi / 2$ and (5.4), we have $L_{\bar{y}_{0}}\left(a_{2}, \bar{y}_{1}\right)>L_{\bar{y}_{0}^{\prime}}\left(a_{2}^{\prime}, \bar{y}_{1}^{\prime}\right)-O\left(\varepsilon^{1 / 2}\right)$. Hence Lemma 5.1(2) implies $d\left(y_{0}, \bar{y}_{1}\right)>$ $d\left(y_{0}^{\prime}, \bar{y}_{1}^{\prime}\right)-O\left(\varepsilon^{1 / 2}\right)$. This together with (5.2) implies

$$
\left|d\left(y_{0}, \bar{y}_{1}\right)-d\left(y_{0}^{\prime}, \bar{y}_{1}^{\prime}\right)\right|<O\left(\varepsilon^{1 / 2}\right) .
$$

Therefore, by Lemma 5.1(1) we see that $L_{\bar{y}_{1}}\left(\bar{y}_{0}, y_{0}\right)<L_{\bar{y}_{1}^{\prime}}\left(\bar{y}_{0}^{\prime}, y_{0}^{\prime}\right)+O\left(\varepsilon^{1 / 4}\right)$. It follows from (5.4) and $\angle_{\bar{y}_{1}^{\prime}}\left(a_{2}^{\prime}, \bar{y}_{0}^{\prime}\right)=\pi / 2$ that

$$
\begin{aligned}
L_{\bar{y}_{1}}\left(y_{0}, y_{1}\right) & \geq L_{\bar{y}_{1}}\left(y_{1}, \bar{y}_{0}\right)-L_{\bar{y}_{1}}\left(\bar{y}_{0}, y_{0}\right) \\
& >\pi / 2-L_{\bar{y}_{1}^{\prime}}\left(\bar{y}_{0}^{\prime}, y_{0}^{\prime}\right)-O\left(\varepsilon^{1 / 4}\right)=L_{\bar{y}_{1}^{\prime}}\left(y_{0}^{\prime}, y_{1}^{\prime}\right)-O\left(\varepsilon^{1 / 4}\right) .
\end{aligned}
$$

By (5.5), (5.6), and Lemma 5.1(2), we have (5.3). This completes the proof. 
5.2. Proof of Theorem 1.7. We need a lemma.

Lemma 5.4. Let $\varepsilon$ and $l$ be positive numbers, and let $c:[0, l] \rightarrow Y$ be a 1-Lipschitz continuous curve from a point $x_{0}$ to a point $x_{1}$ in a metric space $Y$ such that

$$
l<d\left(x_{0}, x_{1}\right)+\varepsilon .
$$

Assume that there exists a minimizing geodesic $\gamma_{x_{0} x_{1}}$ joining $x_{0}$ to $x_{1}$. Then, for any $s \in[0,1]$, setting $x_{s}:=\gamma_{x_{0} x_{1}}\left(s d\left(x_{0}, x_{1}\right)\right)$ we have

$$
d\left(x_{s}, c(s l)\right)<2 \varepsilon .
$$

Note that the parameter of $c$ is not necessarily proportional to the arc-length.

Proof. Since $c$ is 1-Lipschitz continuous, it follows from (5.7) that

$$
d\left(x_{0}, c(s l)\right)+d\left(c(s l), x_{1}\right) \leq s l+(1-s) l<d\left(x_{0}, x_{1}\right)+\varepsilon,
$$

and hence, by the triangle inequality,

$$
0 \leq s l-d\left(x_{0}, c(s l)\right)<\varepsilon .
$$

By $(5.7)$ and $d\left(x_{s}, x_{1}\right) \leq(1-s) l$, we have

$$
s l \geq d\left(x_{0}, x_{s}\right)=d\left(x_{0}, x_{1}\right)-d\left(x_{s}, x_{1}\right)>s l-\varepsilon .
$$

Combining this and (5.8) yields

$$
\left|d\left(x_{0}, x_{s}\right)-d\left(x_{0}, c(s l)\right)\right|<2 \varepsilon .
$$

By the triangle inequality, this completes the proof.

Let $Y$ be a CAT(1)-space with diam $Y \leq \pi / 2$, and let $\rho: Y \rightarrow \mathbb{R}$ be the function defined by $\rho(x):=\sup _{y \in Y} d(x, y)$. By the definition, $\operatorname{rad} Y=\inf _{x \in Y} \rho(x) \leq \pi / 2$. We define the constant $\delta_{m}:=\pi / 2-\operatorname{rad} \triangle_{1}^{m}$, where $\triangle_{1}^{m}$ is the standard spherical simplex defined in (1.1). $\delta_{m}$ is strictly monotone decreasing in $m=1,2, \ldots$ Denote the barycenter of $\triangle_{1}^{m}$ by $b_{m}^{\prime}$.

The distortion dis $\varphi$ of a map $\varphi: A_{1} \rightarrow A_{2}$ between metric spaces is defined by

$$
\operatorname{dis} \varphi:=\sup _{x, y \in A_{1}}|d(\varphi(x), \varphi(y))-d(x, y)| .
$$

We prove the following:

Lemma 5.5. Let $\varepsilon$ be a positive number with $\varepsilon \ll \delta_{m}$. Assume that there exists a 1-Lipschitz continuous map $\varphi_{m}: \triangle_{1}^{m} \rightarrow Y$ such that $\operatorname{dis} \varphi_{m}<\varepsilon$ and $\rho\left(b_{m}\right)>$ $\pi / 2-\varepsilon$, where $b_{m}:=\varphi_{m}\left(b_{m}^{\prime}\right)$. Then, there exists a 1-Lipschitz continuous map $\varphi_{m+1}: \Delta_{1}^{m+1} \rightarrow Y$ such that $\operatorname{dis} \varphi_{m+1}<O\left(\varepsilon^{1 / 8}\right)$. 
Proof. Denote by $a_{0}^{\prime}, \ldots, a_{m+1}^{\prime}$ the vertices of $\triangle_{1}^{m+1}$, and set $a_{i}:=\varphi_{m}\left(a_{i}^{\prime}\right)$. Let $\triangle_{1}^{m} \subset \partial \triangle_{1}^{m+1}$ be the face opposite to $a_{m+1}^{\prime}$. There exists a point $a_{m+1} \in Y$ with $d\left(a_{m+1}, b_{m}\right)>\pi / 2-\varepsilon$. We construct a map $\varphi_{m+1}: \Delta_{1}^{m+1} \rightarrow Y$ as follows. For any given $x^{\prime} \in \triangle_{1}^{m+1}$, the segment $\left[a_{m+1}^{\prime}, x^{\prime}\right]$ extends to a segment $\left[a_{m+1}^{\prime}, \bar{x}^{\prime}\right]$ with $\bar{x}^{\prime} \in \Delta_{1}^{m}$. Set $\bar{x}:=\varphi_{m}\left(\bar{x}^{\prime}\right)$. There is a unique point $x \in\left[a_{m+1}, \bar{x}\right]$ such that

$$
\frac{d\left(a_{m+1}, x\right)}{d\left(a_{m+1}, \bar{x}\right)}=\frac{d\left(a_{m+1}^{\prime}, x^{\prime}\right)}{d\left(a_{m+1}^{\prime}, \bar{x}^{\prime}\right)} .
$$

We then define $\varphi_{m+1}\left(x^{\prime}\right):=x$. It follows that $\varphi_{m+1}\left(a_{m+1}^{\prime}\right)=a_{m+1}$ and $\left.\varphi_{m+1}\right|_{\Delta_{1}^{m}}=$ $\varphi_{m}$. Note that $\varphi_{m}$ and $\varphi_{m+1}$ are not necessarily injective.

Let us prove that for any $z \in \varphi_{m}\left(\triangle_{1}^{m}\right)$,

$$
d\left(a_{m+1}, z\right)>\pi / 2-O\left(\varepsilon^{1 / 2}\right) .
$$

Take a point $z^{\prime} \in \triangle_{1}^{m}$ with $\varphi_{m}\left(z^{\prime}\right)=z$. The segment $\left[b_{m}^{\prime}, z^{\prime}\right]$ extends to a segment $\left[z_{0}^{\prime}, z_{1}^{\prime}\right]$ with $z_{0}^{\prime}, z_{1}^{\prime} \in \partial \triangle_{1}^{m}$. Since $\delta_{m}$ coincides with the radius of the inscribed sphere of $\triangle_{1}^{m}$ centered at $b_{m}^{\prime}$, we have $d\left(b_{m}^{\prime}, z_{i}^{\prime}\right) \geq \delta_{m}$ for each $i=0,1$. Set $z_{i}:=\varphi_{m}\left(z_{i}^{\prime}\right)$. Consider the 1-Lipschitz continuous curve $c:=\varphi_{m} \circ \gamma_{z_{0}^{\prime}}^{\prime} z_{1}^{\prime}$ joining $z_{0}$ and $z_{1}$. Note that $c$ passes through $z$ and $b_{m}$. Choose a number $s \in[0,1]$ with $c\left(s d\left(z_{0}^{\prime}, z_{1}^{\prime}\right)\right)=b_{m}$ and let $b:=\gamma_{z_{0} z_{1}}\left(s d\left(z_{0}, z_{1}\right)\right)$ (cf. Figure 2). Since dis $\varphi_{m}<\varepsilon$,
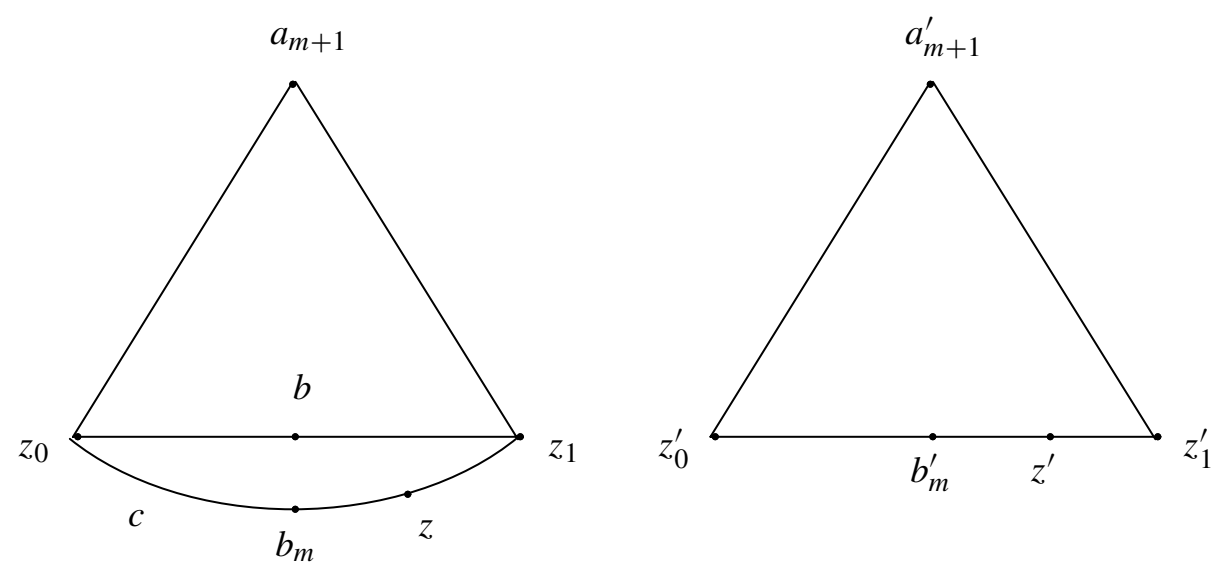

Figure 2. $\Delta\left(z_{0}, z_{1}, a_{m+1}\right)$ and $\Delta\left(z_{0}^{\prime}, z_{1}^{\prime}, a_{m+1}^{\prime}\right)$.

we see that $d\left(z_{0}^{\prime}, z_{1}^{\prime}\right)<d\left(z_{0}, z_{1}\right)+\varepsilon$. Lemma 5.4 implies that $d\left(b, b_{m}\right)<2 \varepsilon$ and so $d\left(a_{m+1}, b\right)>\pi / 2-3 \varepsilon$ by the assumption for $a_{m+1}$. By $\varepsilon \ll \delta_{m}$ we have

$$
d\left(b, z_{i}\right)>d\left(b_{m}^{\prime}, z_{i}^{\prime}\right)-3 \varepsilon \geq \delta_{m}-3 \varepsilon \geq \varepsilon^{1 / 2}
$$


for each $i=0,1$. Applying Lemma 5.2(2) to $\triangle\left(z_{0}, z_{1}, a_{m+1}\right)$ yields that $d\left(a_{m+1}, y\right)>\pi / 2-O\left(\varepsilon^{1 / 2}\right)$ for any $y \in\left[z_{0}, z_{1}\right]$. Therefore, by Lemma 5.4 we obtain (5.9).

For any given two points $x_{0}^{\prime}, x_{1}^{\prime} \in \Delta_{1}^{m+1}$, either segment $\left[a_{m+1}^{\prime}, x_{i}^{\prime}\right]$ extends to a segment $\left[a_{m+1}^{\prime}, \bar{x}_{i}^{\prime}\right]$ with $\bar{x}_{i}^{\prime} \in \triangle_{1}^{m}$. Let $x_{i}:=\varphi_{m+1}\left(x_{i}^{\prime}\right)$ and $\bar{x}_{i}:=\varphi_{m+1}\left(\bar{x}_{i}^{\prime}\right)$. Since $\varphi_{m}$ is 1-Lipschitz continuous, we have $d\left(\bar{x}_{0}, \bar{x}_{1}\right) \leq d\left(\bar{x}_{0}^{\prime}, \bar{x}_{1}^{\prime}\right)$. Comparing $\triangle\left(\bar{x}_{0}, \bar{x}_{1}, a_{m+1}\right)$ and $\triangle\left(\bar{x}_{0}^{\prime}, \bar{x}_{1}^{\prime}, a_{m+1}^{\prime}\right)$, the CAT(1)-inequality leads to $d\left(x_{0}, x_{1}\right) \leq$ $d\left(x_{0}^{\prime}, x_{1}^{\prime}\right)$. Thus, $\varphi_{m+1}$ is 1 -Lipschitz continuous. It remains to prove that

$$
d\left(x_{0}, x_{1}\right)>d\left(x_{0}^{\prime}, x_{1}^{\prime}\right)-O\left(\varepsilon^{1 / 8}\right) .
$$

By Lemma 5.4, for any point $w \in\left[\bar{x}_{0}, \bar{x}_{1}\right]$ there exists a point $z \in \varphi_{m}\left(\left[\bar{x}_{0}^{\prime}, \bar{x}_{1}^{\prime}\right]\right)$ with $d(z, w)<2 \varepsilon$. This and (5.9) imply

$$
d\left(a_{m+1}, w\right)>\pi / 2-O\left(\varepsilon^{1 / 2}\right) .
$$

Consider the small geodesic triangles $\triangle\left(\bar{x}_{0}, \bar{x}_{1}, a_{m+1}\right)$ in $Y$ and $\triangle\left(\bar{x}_{0}^{\prime}, \bar{x}_{1}^{\prime}, a_{m+1}^{\prime}\right)$ in a unit 2-sphere in $\triangle_{1}^{m+1}$. By dis $\varphi_{m}<\varepsilon,(5.11)$, and applying Lemma 5.3 to their triangles, we obtain (5.10). This completes the proof of Lemma 5.5.

Let $n:=\operatorname{dim}_{C} Y+1<\infty$ and let $\varepsilon$ be a positive number with $\varepsilon \ll \delta_{n}$. To prove Theorem 1.7, we suppose that $\operatorname{rad} Y>\pi / 2-\varepsilon$. Note that $\rho(y)>\pi / 2-\varepsilon$ holds for any $y \in Y$. Take a point $a_{0} \in Y$. There exists a point $a_{1} \in Y$ with $d\left(a_{1}, a_{0}\right) \pi / 2-\varepsilon$. Let $\varphi_{1}: \triangle_{1}^{1} \rightarrow\left[a_{0}, a_{1}\right]$ be the linear bijective map. Since $\pi / 2-\varepsilon<d\left(a_{0}, a_{1}\right) \leq \pi / 2$, this is a 1-Lipschitz continuous map $\triangle_{1}^{1} \rightarrow Y$ with dis $\varphi_{1}<\varepsilon$. By Lemma 5.5, we inductively have 1-Lipschitz continuous maps $\varphi_{m}: \triangle_{1}^{m} \rightarrow Y, m=1,2, \ldots, n$, such that $\operatorname{dis} \varphi_{m}<O\left(\varepsilon^{1 / 8^{m}}\right)$. Since $\operatorname{dim} \varphi_{n}\left(\triangle_{1}^{n}\right) \leq \operatorname{dim}_{C} Y=n-1$, Lemma 4.2 implies that $\varphi_{n}$ collapses $\partial \triangle_{1}^{n}$. Hence, there exist $n+1$ points $y_{i}^{\prime} \in F_{i}, i=0,1, \ldots, n$, that are all mapped by $\varphi_{n}$ to a common point of $Y$, where $F_{i} \subset \triangle_{1}^{n}$ is the opposite face to $a_{i}^{\prime}$. We set

$$
\alpha_{n}:=\inf \left\{\max _{i, j} d\left(x_{i}^{\prime}, x_{j}^{\prime}\right) \mid x_{i}^{\prime} \in F_{i}, x_{i}^{\prime} \neq x_{j}^{\prime} \text { for any } i \neq j\right\}>0 .
$$

Then for some $i_{0} \neq j_{0}$ we have $\alpha_{n} \leq d\left(y_{i_{0}}^{\prime}, y_{j_{0}}^{\prime}\right) \leq \operatorname{dis} \varphi_{n}<O\left(\varepsilon^{1 / 8^{n}}\right)$, which gives a lower estimate of $\varepsilon$. Therefore we obtain $\operatorname{rad} Y<\pi / 2-\delta$ for some positive constant $\delta$ depending only on $n$.

Since $Y$ is complete, $C(Y)$ consists of a single point (cf. Proposition 3.1 in [LS2]). This completes the proof of Theorem 1.7.

Remark 5.6. For $A \subset Y$, we denote by $C_{Y}(A)$ the set of all points where the function $Y \ni x \mapsto \sup _{y \in A} d(x, y) \in[0, \infty]$ attains the infimum. For an arbitrary subset $A$ of a CAT(1)-space $Y$ with $\operatorname{diam} A \leq \pi / 2$, we have $\operatorname{diam} A=\operatorname{diam} B$ 
for the closure $B$ of the convex hull of $A$ (cf. Lemma 4.1 in [LS1]). By applying Theorem 1.7 to $B$, we obtain the following generalization. Let $Y$ be a complete CAT(1)-space of $\operatorname{dim}_{C} Y<\infty$, and $A \subset Y$ a subset of $\operatorname{diam} A \leq \pi / 2$. Then $\inf _{x \in Y} \sup _{y \in A} d(x, y)<\pi / 2-\delta$, and $C_{Y}(A)$ consists of a single point.

5.3. Proof of Theorem 1.3. Let $f$ be a parabolic isometry of a proper CAT(0)-space $X$ and let $B:=X_{f}(\infty)$. It follows from Theorem 1.1 and Lemma 3.6(3) that $B$ is a closed, $\pi$-convex subset of $(X(\infty), T d)$ with $\operatorname{rad} B \leq \pi / 2$. Hence, $B$ itself is a complete CAT(1)-space.

First, we verify that $C(B)$ is non-empty. Let $\rho: B \rightarrow \mathbb{R}$ be the function defined by $\rho(x):=\sup _{y \in B} d(x, y)$. There exists a sequence $\left\{x_{i}\right\}$ in $B$ with $\rho\left(x_{i}\right) \rightarrow \operatorname{rad} B$ as $i \rightarrow \infty$. Since $X(\infty)$ is compact with respect to the sphere topology, some subsequence of $\left\{x_{i}\right\}$ converges to a point $x$. We have $x \in B$ because $B$ is closed. By the lower semi-continuity of Tits distances, we have $\rho(x) \leq \operatorname{rad} B$. Thus, $\rho(x)=\operatorname{rad} B$ and $x \in C(B)$.

By the convexity of $B, C(B)$ is a closed, convex subset of $(X(\infty), T d)$ with the property that diam $C(B) \leq \operatorname{rad} B \leq \pi / 2$. By setting $Y:=C(B)$, it is a complete CAT(1)-space of diam $Y \leq \pi / 2$. By Proposition 1.8, $\operatorname{dim}_{C}(X(\infty), T d)$ is finite. Therefore, by Theorem 1.7 we have $\operatorname{rad} Y<\pi / 2$, and $C(Y)$ consists of a single point. Moreover, the second half follows from the uniqueness of the point and its property. This completes the proof of Theorem 1.3.

Theorem 1.7 and the proof of Theorem 1.3 imply the following:

Proposition 5.7. Let $Y$ be a compact CAT(1)-space of $\operatorname{dim} Y<\infty$ and $\operatorname{rad} Y \leq \pi / 2$. Then $C^{2}(Y)$ consists of a single point.

Remark 5.8. Let $X$ be a complete CAT(0)-space and $G$ a subgroup of the isometry group of $X$. Set $X_{G}(\infty):=\bigcap\left\{X_{g}(\infty) \mid g \in G\right\}$. We say that $G$ is admissible if $X_{G}(\infty) \neq \emptyset$ and $\operatorname{rad} X_{G}(\infty) \leq \pi / 2$. It follows from Theorem 1.3 that if $G$ is an abelian group containing a parabolic element, then $G$ is admissible, provided $X$ is as in Theorem 1.3. This is an extension of Proposition 4.4.2 of [E]. Similarly, we can obtain some extensions of Propositions 4.4.3, 4.4.4, and Corollary 4.4.5 of [E] for CAT(0)-spaces. Proposition 4.4.6 of [E] can be also extended by using the flat torus theorem for CAT(0)-spaces (cf. Theorem II.7.1 of [BH]).

\section{Example of a symmetric space}

In this section we discuss the symmetric space $\operatorname{SL}(3, \mathbb{R}) / \operatorname{SO}(3, \mathbb{R})$ in detail as an example for Theorems 1.1 and 1.3. A good reference for standard facts we use here is II.10 in [BH]. We would like to thank M. Bestvina for suggesting this example, and also K. Wortman for useful discussions and informations. 
6.1. Manifolds $\boldsymbol{P}(\boldsymbol{n}, \mathbb{R})$ and $\boldsymbol{P}(\boldsymbol{n}, \mathbb{R})_{1}$. Let $P(n, \mathbb{R})$ denote the space consisting of all positive definite, symmetric $(n \times n)$-matrices with real coefficients. Naturally, $P(n, \mathbb{R})$ is a differentiable manifold of dimension $n(n+1) / 2$. The tangent space $T_{p} P(n, \mathbb{R})$ at a point $p$ is naturally isomorphic (via translation) to the space of all symmetric $(n \times n)$ - matrices, $S(n, \mathbb{R})$. The inner product $(u, v)_{p}=\operatorname{tr}\left(p^{-1} u p^{-1} v\right)$ on $T_{p} P(n, \mathbb{R}) \simeq S(n, \mathbb{R})$ defines a Riemannian metric on $P(n, \mathbb{R})$, where tr $u$ is the trace of a matrix $u$. $P(n, \mathbb{R})$ is a simply connected, complete, non-positively curved Riemannian manifold, so that it is a proper CAT( 0$)$-space.

Let $P(n, \mathbb{R})_{1} \subset P(n, \mathbb{R})$ be the subset of matrices with determinant $1 . P(n, \mathbb{R})_{1}$ is a totally geodesic submanifold, whose tangent space at $p$ is the subspace in $S(n, \mathbb{R})$ of matrices with trace $0 . P(n, \mathbb{R})$ is a simply connected, complete, non-positively curved Riemannian manifold of dimension $n(n+1) / 2-1$, so that it is a proper CAT(0)-space as well.

$\mathrm{SL}(n, \mathbb{R})$ acts on $P(n, \mathbb{R})$ by isometries according to the rule

$$
f(p):=f p^{t} f, \quad p \in P(n, \mathbb{R}), f \in \operatorname{SL}(n, \mathbb{R}),
$$

where ${ }^{t} f$ is the transpose of $f$. The right hand side of the definition is by the multiplication of matrices. We may write $f \cdot p$ instead of $f(p) . P(n, \mathbb{R})_{1}$ is invariant by this action, and the action is transitive on this submanifold. Let $e$ be the identity matrix. The stabilizer of $e$ is $\mathrm{SO}(n)$, so that $P(n, \mathbb{R})_{1}$ is identified as $\mathrm{SL}(n, \mathbb{R}) / \mathrm{SO}(n)$.

6.2. Geometry of $P(3, \mathbb{R})_{1}$ and Tits boundary. We collect some standard facts on $P(3, \mathbb{R})_{1}$ from II.10 in $[\mathrm{BH}]$. Most of them are true for all $P(n, \mathbb{R})_{1}, n \geq 3$ with appropriate change. Put $X:=P(3, \mathbb{R})_{1} . X$ is a 5-dimensional, irreducible symmetric space of non-compact type of rank 2 , which is a proper CAT(0)-space.

Let us denote the Tits boundary $(X(\infty), T d)$ by $X(\infty)$ for simplicity. $X(\infty)$ is a "thick spherical building" of dimension 1 such that each apartment is isometric to $\mathbb{S}^{1}(1)$ and each Weyl chamber at infinity is an edge of length $\pi / 3$. Moreover, diam $X(\infty)=\pi$. Since $X(\infty)$ is a spherical building, any two Weyl chambers at infinity are contained in at least one apartment.

The action of $\operatorname{SL}(3, \mathbb{R})$ induced on $X(\infty)$ is by simplicial isometries. It is transitive on pairs $(A, c)$, where $A$ is an apartment, and $c \subset A$ is a Weyl chamber at infinity. A Weyl chamber is a fundamental domain for the action. (cf. II.10.71, 75, $76,77$ in $[\mathrm{BH}])$. Therefore there are two orbits in the vertices of $X(\infty)$ by the group action, so that $X(\infty)$ is a bi-partite graph. It follows that any loop in $X(\infty)$ consists of an even number of edges.

The isometry group of $X, I(X)$, has two connected components, and the one which contains the identity map, $I_{0}(X)$, is $\operatorname{SL}(3, \mathbb{R})$. Let $\sigma$ be the involution of $X$ at $e$, which is an orientation reversing isometry. It is given by $\sigma(f)={ }^{t} f^{-1}$. $I(X)=I_{0}(X) \cup \sigma I_{0}(X)$. 
Let $f$ be an isometry of $X$. $\operatorname{Min}(f)$ denotes the set of all points in $X$ at which the displacement function $d_{f}$ of $f$ attains its infimum $|f|:=\inf _{p \in X} d_{f}(p)$, which is the translation length. If $f$ is elliptic, then $\operatorname{Min}(f)$ coincides with the fixed point set Fix $(f)$ of $f$ in $X$. If $f$ is hyperbolic, then the axes of $f$ are parallel to each other, and the union of their images is $\operatorname{Min}(f)$. If $f$ is parabolic, then $\operatorname{Min}(f)=\emptyset . f$ is said to be semi-simple if $f$ is elliptic or hyperbolic.

In this section we calculate those geometric characters of $f \in \operatorname{SL}(3, \mathbb{R})$.

6.3. Real Jordan forms. It is known that $f \in \mathrm{SL}(3, \mathbb{R})$ is semi-simple as an isometry of $X$ if and only if it is semi-simple as a matrix, i.e., diagonalizable in $\operatorname{GL}(3, \mathbb{C})$. (cf. II.10.61 in $[\mathrm{BH}])$.

Calculation of $X_{f}(\infty)$ and $\operatorname{Min}(f)$ of $f \in \operatorname{SL}(3, \mathbb{R})$ is mostly by linear algebra. Each $f \in \operatorname{SL}(3, \mathbb{R})$ is conjugate to $g$ in $\operatorname{SL}(3, \mathbb{R})$ such that $g$ is one (and only one) of the following list. $g$ is a real Jordan form of $f$. The symbol $\operatorname{diag}(a, b, c)$ is for the $(3 \times 3)$-diagonal matrix with entries $a, b, c$.

Since $f$ and $g$ are conjugate in $I(X), f$ is elliptic, hyperbolic, or parabolic if and only if so is $g$, respectively. If $h \in I(X)$ is a conjugating element, i.e., $h f h^{-1}=g$, then $X_{f}(\infty)=h \cdot X_{g}(\infty), \operatorname{Min}(f)=h \cdot \operatorname{Min}(g)$, and $|f|=|g|$. We discuss $g$ instead of $f$.

\section{List of real Jordan forms in $\operatorname{SL}(3, \mathbb{R})$.}

$$
\begin{aligned}
& \text { (1) }\left(\begin{array}{lll}
1 & 0 & 0 \\
0 & 1 & 1 \\
0 & 0 & 1
\end{array}\right) \text {. } \\
& \text { (2) }\left(\begin{array}{ccc}
1 / a^{2} & 0 & 0 \\
0 & a & 1 \\
0 & 0 & a
\end{array}\right) \text {, where } 0,1 \neq a \in \mathbb{R} \text {. } \\
& \text { (3) }\left(\begin{array}{ccc}
1 & 1 & 0 \\
0 & 1 & 1 \\
0 & 0 & 1
\end{array}\right) \text {. } \\
& \text { (4) }\left(\begin{array}{ccc}
a & b & 0 \\
-b & a & 0 \\
0 & 0 & 1 /\left(a^{2}+b^{2}\right)
\end{array}\right) \text { where } a, b \in \mathbb{R} \text { with } a^{2}+b^{2} \neq 0 \text { and } b \neq 0 .
\end{aligned}
$$

This one is conjugate to $\operatorname{diag}\left(a+i b, a-i b, 1 /\left(a^{2}+b^{2}\right)\right)$ by an element in $\mathrm{GL}(3, \mathbb{C})$.

(5) $\operatorname{diag}(a, b, c)$ such that $a, b, c \in \mathbb{R}, a b c=1, a \neq b \neq c \neq a$.

(6) $\operatorname{diag}\left(a, a, 1 / a^{2}\right), a \in \mathbb{R}, a \neq 0,1$.

(7) $e$. 
6.4. Flat and Weyl chambers. Consider the following linear subspace in $T_{e} X$.

$$
\mathfrak{a}_{0}:=\left\{u \mid u=\operatorname{diag}\left(u_{1}, u_{2}, u_{3}\right), \operatorname{tr} u=0\right\} \subset T_{e} X .
$$

Let

$$
F_{0}:=\left\{\exp (u) \mid u \in \mathfrak{a}_{0}\right\} \subset X .
$$

$F_{0}$ is a flat plane in $X$ and $A_{0}:=F_{0}(\infty)$ is an apartment in $X(\infty)$.

For $x \in A_{0}$, let $\gamma_{e x}$ be the geodesic in $F_{0}$ from $e$ to $x \cdot \gamma_{e x}$ is $\exp (t u(x)), t \geq 0$ for some $u(x) \in \mathfrak{a}_{0}$. The tangent vector at $e, u(x)$, is uniquely determined by $x$ up to scaling by a positive number, so that let us denote the one of unit length by $u(x)$, which we write as $\operatorname{diag}\left(u_{1}(x), u_{2}(x), u_{3}(x)\right)$.

$A_{0}$ is a 6-gon as a building with the following Weyl chambers (see Figure 3 ). $\left\{u_{i}(x) \geq u_{j}(x) \geq u_{k}(x)\right\}$ means the set $\left\{x \in A_{0} \mid u_{i}(x) \geq u_{j}(x) \geq u_{k}(x)\right\}$.

$$
\begin{array}{ll}
c_{1}:=\left\{u_{1}(x) \geq u_{2}(x) \geq u_{3}(x)\right\}, & c_{2}:=\left\{u_{2}(x) \geq u_{1}(x) \geq u_{3}(x)\right\}, \\
c_{3}:=\left\{u_{2}(x) \geq u_{3}(x) \geq u_{1}(x)\right\}, & c_{4}:=\left\{u_{3}(x) \geq u_{2}(x) \geq u_{1}(x)\right\}, \\
c_{5}:=\left\{u_{3}(x) \geq u_{1}(x) \geq u_{2}(x)\right\}, & c_{6}:=\left\{u_{1}(x) \geq u_{3}(x) \geq u_{2}(x)\right\} .
\end{array}
$$

$A_{0}=c_{1} \cup \cdots \cup c_{6}$. Define $v_{i}:=c_{i-1} \cap c_{i}, i=1,2, \ldots, 6$, where $c_{0}=c_{6}$. They are the vertices of $X(\infty)$ in $A_{0}$. We may write $c_{i}=\left[v_{i}, v_{i+1}\right], 1 \leq i \leq 6$, where $v_{7}=v_{1}$.

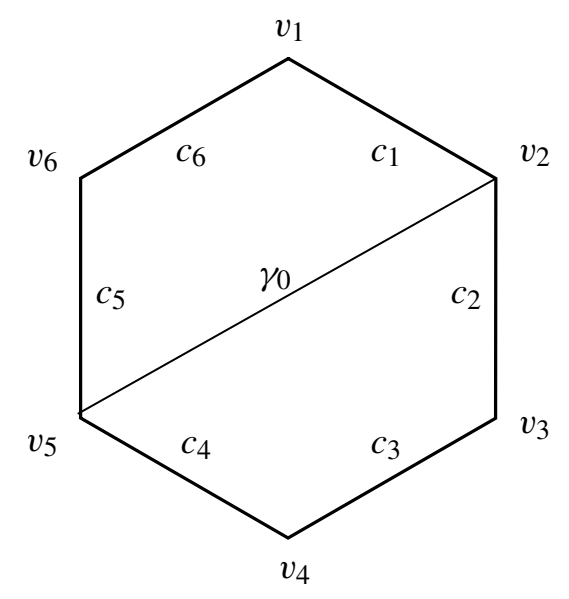

Figure 3. 6-gon.

A bi-infinite geodesic, or simply line, in $X$ is always contained in some flat plane because $X$ is a symmetric space. If a line is contained in a unique flat, then it is called regular (cf. $10.46[\mathrm{BH}]$ ), and otherwise it is called singular. There are three 
singular bi-infinite geodesics (without orientation) on $F_{0}$, which are $\gamma_{e v_{1}} \cup \gamma_{e v_{4}}, \gamma_{e v_{2}} \cup$ $\gamma_{e v_{5}}, \gamma_{e v_{3}} \cup \gamma_{e v_{6}}$.

Set $w_{2}:=u\left(v_{2}\right)=(1 / \sqrt{6}) \operatorname{diag}(1,1,-2)$. This is a unit vector at $e$ tangent to $F_{0}$, pointing the vertex $v_{2}$ at infinity. Define a line in $F_{0}$ by

$$
\gamma_{0}(t):=\exp \left(t w_{2}\right), \quad t \in \mathbb{R} .
$$

$\gamma_{0}$ is a line through $e$ such that $\gamma_{0}(\infty)=v_{2}, \gamma_{0}(-\infty)=v_{5}$. As a set, $\gamma_{0}=$ $\left\{\operatorname{diag}\left(s, s, 1 / s^{2}\right) \mid 0<s \in \mathbb{R}\right\}=\gamma_{e v_{2}} \cup \gamma_{e v_{5}} \cdot \gamma_{0}$ is a singular geodesic.

For a line $\gamma$ in $X$, let $P(\gamma)$ denote the union of all lines in $X$ parallel to $\gamma$. This is a convex subset in $X$, so that let $P(\gamma)(\infty) \subset X(\infty)$ denote the set of points at infinity of $P(\gamma)$.

Denote by $\mathcal{F}_{0}$ the set of all flat planes in $X$ containing $\gamma_{0}$. Then, $P\left(\gamma_{0}\right)=\bigcup\{F \mid$ $\left.F \in \mathcal{F}_{0}\right\} . P\left(\gamma_{0}\right)$ is a totally geodesic, 3-dimensional submanifold, which is naturally isometric to $P(2, \mathbb{R})_{1} \times \mathbb{R}$ (cf. Proposition II.10.67 in $\left.[\mathrm{BH}]\right) . P(2, \mathbb{R})_{1}$ is isometric to $\mathbb{H}^{2}$ up to a scaling factor. We note that $P\left(\gamma_{0}\right)(\infty)=\bigcup\left\{F(\infty) \mid F \in \mathcal{F}_{0}\right\}$.

\subsection{Theorem}

Theorem 6.1. Suppose $g \in \mathrm{SL}(3, \mathbb{R})$ is one in the list of Subsection 6.3. Then we have the following in the order of the list:

(1) $g$ is parabolic and $X_{g}(\infty)$ is the union of all edges incident to $c_{2} . X_{g}(\infty)$ is not compact in $(X(\infty), T d)$, with uncountably many edges; $|g|=0$.

(2) $g$ is parabolic and $X_{g}(\infty)=c_{1} \cup c_{2} \cup c_{3} ;|g|=2 \sqrt{6} \log |a|$.

(3) $g$ is parabolic and $X_{g}(\infty)=c_{1} ;|g|=0$.

(4) $g$ is semi-simple, and

(a) $X_{g}(\infty)=\left\{v_{2}, v_{5}\right\}$.

(b) If $a^{2}+b^{2}=1$, then $g$ is elliptic, and $\operatorname{Fix}(g)=\gamma_{0}$.

(c) If $a^{2}+b^{2} \neq 1$, then $g$ is hyperbolic and $|g|=\sqrt{6} \log \left(a^{2}+b^{2}\right)$; $\operatorname{Min}(g)=\gamma_{0}$

(5) $g$ is hyperbolic, and $|g|=2 \sqrt{(\log |a|)^{2}+(\log |b|)^{2}+(\log |c|)^{2}}$.

(a) $X_{g}(\infty)=A_{0}$.

(b) $\operatorname{Min}(g)=F_{0}$.

(6) $g$ is hyperbolic, and $|g|=2 \sqrt{6} \log |a|$.

(a) $X_{g}(\infty)=P\left(\gamma_{0}\right)(\infty)$.

(b) $\operatorname{Min}(g)=P\left(\gamma_{0}\right)$.

(7) $g$ is the identity map, i.e. elliptic, with $\operatorname{Fix}(g)=X$ and $X_{g}(\infty)=X(\infty)$. 
6.6. Stabilizers. The analysis of the stabilizing subgroup in $\operatorname{SL}(3, \mathbb{R})$ of a point $v \in X(\infty)$ is important for the proof of the theorem. We quote Proposition II.10.64 in $[\mathrm{BH}]$ in the following form.

Lemma 6.2. Let $g=\left(g_{i j}\right) \in \operatorname{SL}(3, \mathbb{R})$, and $x \in A_{0}$. Then $g(x)=x$ if and only if $g_{i j} e^{-t\left(u_{i}(x)-u_{j}(x)\right)}$ converges as $t \rightarrow \infty$ for all $i, j$.

This implies the following.

Proposition 6.3. Let $G_{i}$ be the subgroups of $\operatorname{SL}(3, \mathbb{R})$ stabilizing $v_{i}$. Then,

$$
\begin{aligned}
& G_{1}=\left\{\left(\begin{array}{lll}
* & * & * \\
0 & * & * \\
0 & * & *
\end{array}\right)\right\}, \quad G_{2}=\left\{\left(\begin{array}{lll}
* & * & * \\
* & * & * \\
0 & 0 & *
\end{array}\right)\right\}, \quad G_{3}=\left\{\left(\begin{array}{ccc}
* & 0 & * \\
* & * & * \\
* & 0 & *
\end{array}\right)\right\} \text {, } \\
& G_{4}=\left\{\left(\begin{array}{ccc}
* & 0 & 0 \\
* & * & * \\
* & * & *
\end{array}\right)\right\}, \quad G_{5}=\left\{\left(\begin{array}{lll}
* & * & 0 \\
* & * & 0 \\
* & * & *
\end{array}\right)\right\}, \quad G_{6}=\left\{\left(\begin{array}{ccc}
* & * & * \\
0 & * & 0 \\
* & * & *
\end{array}\right)\right\} \text {, }
\end{aligned}
$$

where $* \in \mathbb{R}$.

Let $H_{1}$ be the following subgroup, parameterized by $t \in \mathbb{R}$, which fixes edges $c_{1}$, $c_{2}, c_{3}$, pointwise.

$$
H_{1}=\left\{\left(\begin{array}{lll}
1 & 0 & 0 \\
0 & 1 & t \\
0 & 0 & 1
\end{array}\right) \mid t \in \mathbb{R}\right\} .
$$

$H_{1}$ fixes $v_{1} \in X(\infty)$. $H_{1}$ acts transitively on the set of all edges incident to $v_{1}$ other than $c_{1}$. To see it, consider the following subgroup in $\operatorname{SL}(3, \mathbb{R})$ containing $H_{1}$.

$$
J_{1}=\left\{\left(\begin{array}{ccc}
* & 0 & 0 \\
0 & * & * \\
0 & 0 & *
\end{array}\right) \mid * \in \mathbb{R}\right\} .
$$

For a given edge $c \neq c_{1}$, incident to $v_{1}$, we will find $h \in H_{1}$ with $h(c)=c_{6}$. Take an apartment, $A$, containing $c$ and $c_{3}$. Then it automatically contains $c_{1}, c_{2}$ as well. Recall that $\operatorname{SL}(3, \mathbb{R})$ acts transitively on the set of pairs of an apartment, $A^{\prime}$, in $X(\infty)$ and a Weyl chamber, $c^{\prime}$, in $A^{\prime},\left(A^{\prime}, c^{\prime}\right)$. Take $j \in \operatorname{SL}(3, \mathbb{R})$ which maps $(A, c)$ to $\left(A_{0}, c_{6}\right)$. Clear that $j \in J_{1}$ since it fixes $c_{1}, c_{2}, c_{3}$. Let $j=\left(\begin{array}{ccc}p & 0 & 0 \\ 0 & q & s \\ 0 & 0 & r\end{array}\right), p q r=1$. Take $k=\left(\begin{array}{ccc}1 / p & 0 & 0 \\ 0 & 1 / q & 0 \\ 0 & 0 & 1 / r\end{array}\right) \in \operatorname{SL}(3, \mathbb{R})$. Then $k j=\left(\begin{array}{ccc}1 & 0 & 0 \\ 0 & 1 & s / q \\ 0 & 0 & 1\end{array}\right)=h \in H_{1}$. We have $k\left(c_{i}\right)=c_{i}$ for all $i$, so it follows from $j(c)=c_{6}$ that $h(c)=k j(c)=k\left(c_{6}\right)=c_{6}$. 
Also, $H_{1}$ acts transitively on the set of all edges incident at $v_{4}$ other than $c_{3}$. Each of the two sets is parameterized by $t$ by the action.

Off-diagonal entries except for the $(2,3)$-entries of matrices in $H_{1}$ are 0 . Since there are 6 off-diagonal entries in $(3 \times 3)$-matrices, we consider 5 other similar subgroups, $\mathrm{H}_{2}, \mathrm{H}_{3}, \mathrm{H}_{4}, \mathrm{H}_{5}, \mathrm{H}_{6}$, which we define later.

6.7. Proof. We discuss each case in the order and prove Theorem 6.1. Case 7 is trivial.

Case 1. $g$ is parabolic since it is not diagonalizable as a matrix. By Proposition 6.3, $X_{g}(\infty) \cap A_{0}=c_{1} \cup c_{2} \cup c_{3}$. To see that any edge, $c \neq c_{2}, c_{3}$, incident to $v_{3}$ is fixed by $g$, take a (unique) element $h \in H_{6}$ such that $h(c)=c_{3}$, where

$$
H_{6}=\left\{\left(\begin{array}{lll}
1 & 0 & t \\
0 & 1 & 0 \\
0 & 0 & 1
\end{array}\right) \mid t \in \mathbb{R}\right\} .
$$

Since $g\left(c_{3}\right)=c_{3}, h^{-1} g h(c)=c$. Then it follows from $h g=g h$ that $g(c)=c$, pointwise.

To see any edge $c \neq c_{2}$, incident to $v_{2}$ is fixed by $g$, take a (unique) element $h \in H_{2}$ such that $h(c)=c_{1}$, where

$$
H_{2}=\left\{\left(\begin{array}{lll}
1 & 0 & 0 \\
t & 1 & 0 \\
0 & 0 & 1
\end{array}\right) \mid t \in \mathbb{R}\right\} .
$$

As before, we have $h g=g h$, therefore $g(c)=c$, pointwise. We know $X_{g}(\infty)$ has no more edges because it is connected and its diameter is at most $\pi$.

The edges in $X_{g}(\infty)$ other than $c_{1}, c_{2}, c_{3}$ are those which are parametrized by $H_{2}$ and the others which are parametrized by $H_{6}$, so that uncountable. It is not compact because the mid points of the edges are at least $\pi / 3$ apart from each other.

Let us prove $|g|=0$ by computation. To deal with Case 3 at one time, suppose $g=\left(\begin{array}{lll}1 & k & 0 \\ 0 & 1 & 1 \\ 0 & 0 & 1\end{array}\right)$. It suffices for us to show that there exists a geodesic, $\gamma(t)$, such that $\lim _{t \rightarrow \infty} d(g(\gamma(t)), \gamma(t))=0$. We use the notations from the subsection 6.4. For $x \in A_{0}$, set $\gamma(t)=\gamma_{e x}(t)=\exp (t u)$, where $u$ is the diagonal matrix $u(x)=$ $\operatorname{diag}\left(u_{1}, u_{2}, u_{3}\right)$. For simplicity, we write the result of the action by a group element $g$ on a point $p$ as $g \cdot p$, instead of $g(p)$, in this discussion. Then,

$$
\begin{aligned}
d(g \cdot \exp (t u), \exp (t u)) & =d\left(\exp \left(-\frac{t}{2} u\right) \cdot g \cdot \exp (t u), \exp \left(-\frac{t}{2} u\right) \cdot \exp (t u)\right) \\
& =d\left(\exp \left(-\frac{t}{2} u\right) g \exp \left(\frac{t}{2} u\right) \cdot e, e\right)
\end{aligned}
$$


because $\exp \left(\frac{t}{2} u\right) \cdot e=\exp (t u)$. By the computation of matrix multiplications, $\exp \left(-\frac{t}{2} u\right) g \exp \left(\frac{t}{2} u\right)$ is

$$
\left(\begin{array}{ccc}
1 & k \exp \left(t\left(u_{2}-u_{1}\right) / 2\right) & 0 \\
0 & 1 & \exp \left(t\left(u_{3}-u_{2}\right) / 2\right) \\
0 & 0 & 1
\end{array}\right) .
$$

For $g$ in Case 1, we have $k=0$, so that if $u_{2}>u_{3}$, then as $t \rightarrow \infty$, this matrix tends to $e$, which means that $d(g \cdot \gamma(t), \gamma(t)) \rightarrow 0$. We got $|g|=0$. We remark that $u_{2}>u_{3}$ is satisfied for $x \in A_{0}$ if and only if $x \in\left(c_{1} \cup c_{2} \cup c_{3}\right) \backslash\left(v_{1} \cup v_{4}\right)$.

For $g$ in Case 3, we have $k=1$. So, if $u_{1}>u_{2}>u_{3}$, then $d(g \cdot \gamma(t), \gamma(t)) \rightarrow 0$, which shows that $|g|=0$. The condition $u_{1}>u_{2}>u_{3}$ holds for $x \in A_{0}$ if and only if $x \in c_{1} \backslash\left(v_{1} \cup v_{2}\right)$.

Case 2. As in Case 1, $g$ is parabolic and $X_{g}(\infty) \cap A_{0}=c_{1} \cup c_{2} \cup c_{3}$. To see there are not more edges than those in $X_{g}(\infty)$, suppose there was an edge, $c \neq c_{2}, c_{3}$, incident to $v_{3}$ with $g(c)=c$. Take, as before, $h \in H_{6}$ such that $h(c)=c_{3}$.

$$
h=\left(\begin{array}{lll}
1 & 0 & t \\
0 & 1 & 0 \\
0 & 0 & 1
\end{array}\right)
$$

Then, $h g h^{-1}\left(c_{3}\right)=c_{3}$. Since $c \neq c_{3}$, we have $t \neq 0$, which is important to get a contradiction in this case. By computation

$$
h g h^{-1}=\left(\begin{array}{ccc}
1 / a^{2} & 0 & t\left(a-1 / a^{2}\right) \\
0 & a & 1 \\
0 & 0 & a
\end{array}\right) .
$$

Since $t\left(a-1 / a^{2}\right) \neq 0, h g h^{-1}$ is not in $G_{4}$, so that does not fix $v_{4} \in c_{3}$, a contradiction.

To see there is no edge $c \neq c_{1}, c_{2}$ at $v_{2}$ with $g(c)=c$, use $H_{2}$, as before. If there was, take $h \in H_{2}$ with $h(c)=c_{1}$ such that

$$
h=\left(\begin{array}{lll}
1 & 0 & 0 \\
t & 1 & 0 \\
0 & 0 & 1
\end{array}\right), \quad t \neq 0
$$

Then $h g h^{-1}\left(c_{1}\right)=c_{1}$, pointwise. By computation,

$$
h g h^{-1}=\left(\begin{array}{ccc}
1 / a^{2} & 0 & 0 \\
t\left(1 / a^{2}-a\right) & a & 1 \\
0 & 0 & a
\end{array}\right)
$$

such that $t\left(1 / a^{2}-a\right) \neq 0$, therefore $h g h^{-1} \notin G_{1}$ does not fix $v_{1}$, which gives a contradiction since it is supposed to fix $c_{1}=\left[v_{1}, v_{2}\right]$. 
We get the claim because $X_{g}(\infty)$ is connected and with diameter at most $\pi$.

We postpone the computation of $|g|$ until the proof of Case 6. It is not a coincidence that $|g|$ is the same number in Cases 2 and 6.

Case 3. As in Case 1, $g$ is parabolic and $X_{g}(\infty) \cap A_{0}=c_{1}$. To see this is all, suppose there was an edge, $c \neq c_{1}$, incident to $v_{1}$ with $g(c)=c$. Take $h \in H_{1}$ such that $h(c)=c_{6}$. It follows that $h g h^{-1}\left(c_{6}\right)=c_{6}$. Let

$$
h=\left(\begin{array}{lll}
1 & 0 & 0 \\
0 & 1 & t \\
0 & 0 & 1
\end{array}\right)
$$

By computation

$$
h g h^{-1}=\left(\begin{array}{ccc}
1 & 1 & -t \\
0 & 1 & 1 \\
0 & 0 & 1
\end{array}\right),
$$

therefore $h g h^{-1} \notin G_{6}$ does not fix $v_{6} \in c_{6}$, a contradiction.

To see $g$ does not fix any edge incident to $v_{2}$ other than $c_{1}$, use $H_{5}$ and do the same argument, where

$$
H_{5}=\left\{\left(\begin{array}{lll}
1 & t & 0 \\
0 & 1 & 0 \\
0 & 0 & 1
\end{array}\right) \mid t \in \mathbb{R}\right\}
$$

We get the claim since $X_{g}(\infty)$ is connected.

Since we already showed that $|g|=0$ in the proof of Case 1, we finish Case 3 .

Case 4. $g$ is semi-simple because it is diagonalizable in $\operatorname{GL}(3, \mathbb{C})$.

(a) By Proposition 6.3, $X_{g}(\infty) \cap A_{0}=\left\{v_{2}, v_{5}\right\}$. To see this is all, we first show that there is no edge incident to $v_{2}$ in $X_{g}(\infty)$. Suppose there was one, $c$. We know that $c \neq c_{1}, c_{2}$. Take $h \in H_{2}$ such that $h(c)=c_{1}$. If $h=\left(\begin{array}{lll}1 & 0 & 0 \\ t & 1 & 0 \\ 0 & 0 & 1\end{array}\right)$, then

$$
h g h^{-1}=\left(\begin{array}{ccc}
a-t b & b & 0 \\
-b\left(1+t^{2}\right) & a+t b & 0 \\
0 & 0 & 1 /\left(a^{2}+b^{2}\right)
\end{array}\right) \text {, }
$$

which does not fix $v_{1}$ because $-b\left(1+t^{2}\right) \neq 0$. But $h g h^{-1}\left(c_{1}\right)=h g(c)=h(c)=c_{1}$, so that it fixes $v_{1} \in c_{1}$, a contradiction. Similarly there is no edge in $X_{g}(\infty)$ incident to $v_{5}$.

To finish, suppose there was a vertex, $v$, in $X_{g}(\infty) \backslash A_{0}$. Then $\operatorname{Td}\left(v, v_{2}\right)=\pi$, because if it was less than $\pi$, then the unique geodesic from $v$ to $v_{2}$ would have to be in $X_{g}(\infty)$, which is impossible since there is no edge incident to $v_{2}$ fixed by $g$. By the same reason, $\operatorname{Td}\left(v, v_{5}\right)=\pi$. Consider a loop made of three geodesics: one from 
$v$ to $v_{2}$, one from $v_{2}$ to $v_{5}$ and one from $v_{5}$ to $v$. This loop consists of 9 edges, which is impossible because $X(\infty)$ is a bi-partite graph.

(b) Recall that we have $\gamma_{0}=\left\{\operatorname{diag}\left(s, s, 1 / s^{2}\right) \mid 0<s \in \mathbb{R}\right\}$. Under the condition $a^{2}+b^{2}=1$, by computation, $g p^{t} g=p, p \in P(3, \mathbb{R})_{1}$ if and only if $p=\operatorname{diag}\left(s, s, 1 / s^{2}\right), 0<s \in \mathbb{R}$. We get the claim.

(c). By computation, $g\left(\gamma_{0}\right)=\gamma_{0}$. Since $e \in \gamma_{0}$ and $e \neq g(e), g$ is hyperbolic, and $\gamma_{0}$ is an axis. The translation length, $|g|$, is $d(e, g(e))=d\left(e, g e^{t} g\right)=d\left(e, g^{t} g\right)$, where

$$
g^{t} g=\operatorname{diag}\left(a^{2}+b^{2}, a^{2}+b^{2}, 1 /\left(a^{2}+b^{2}\right)^{2}\right)=\exp \left[\log \left(a^{2}+b^{2}\right) \operatorname{diag}(1,1,-2)\right] .
$$

Since the norm $|\operatorname{diag}(1,1,-2)|_{e}=\sqrt{6},|g|=\sqrt{6} \log \left(a^{2}+b^{2}\right)$.

We know that $\gamma_{0} \subset \operatorname{Min}(g) \subset P\left(\gamma_{0}\right)$ because an axis of $g$ is parallel to $\gamma_{0}$. Since $g$ leaves $\gamma_{0}$ invariant, $g$ leaves $P\left(\gamma_{0}\right)$ invariant as well. We remark that the action of $g$ is by a shift and a rotation about $\gamma_{0}$. We define the following subgroup in $\operatorname{SL}(3, \mathbb{R})$, which is in fact in $\mathrm{SO}(3)$.

$$
\begin{array}{r}
R=\left\{h=\left(h_{i j}\right) \in \mathrm{SO}(3) \mid\left(h_{i j}\right)_{1 \leq i, j \leq 2} \in \mathrm{SO}(2), h_{33}=1,\right. \\
\left.h_{13}=h_{31}=h_{23}=h_{32}=0\right\} .
\end{array}
$$

If $h \in R, h$ fixes $e, v_{2}, v_{5}$, so that $h$ fixes all points on $\gamma_{0}$. Therefore $h$ leaves $P\left(\gamma_{0}\right)$ invariant, and acts on $\mathscr{F}_{0}$.

Claim. The action of $R$ on $\mathscr{F}_{0}$ is transitive.

To see it, let $F \in \mathcal{F}_{0}$ be a flat. Then there is an element $w \in T_{e} X$ such that $w$ and $w_{2}$ commute as matrices and the image by exp of the subspace spanned by $w, w_{2}$ in $T_{e} X$ is $F$. The two commuting symmetric matrices $w, w_{2}$ are simultaneously diagonalizable by an element, $h$, in $\mathrm{SO}(3)$. Moreover since $w_{2}$ is diagonal, one may assume that $h$ commutes with $w_{2}$. By computation, this implies that $h$ is in $R$. We claim that $h$ maps $F$ to $F_{0}$. Indeed, let $\gamma$ be the geodesic through $e$ defined by $\gamma=\exp (s w), s \in \mathbb{R}$. It is in $F$. Since $h\left(\gamma_{0}\right)=\gamma_{0}$, it suffices to show $h(\gamma) \subset F_{0}$. Since $h \in \mathrm{SO}(3)$

$$
h(\gamma)=h \exp (s w)^{t} h=h \exp (s w) h^{-1}=\exp \left(s h w h^{-1}\right),
$$

which is in $F_{0}$ because $h w h^{-1}$ is diagonal. We got the claim.

Suppose there was an axis of $g, \alpha$, which is not $\gamma_{0}$. Take the plane $F \in \mathcal{F}_{0}$ which contains $\alpha$. Such $F$ exists since $\alpha$ is parallel to $\gamma_{0}$. Take $h \in R$ with $h(F)=F_{0}$. Since $h$ commutes with $g, h(\alpha)$ is an axis of $g$ as well. It implies that $F_{0}$ is invariant by $g$, so that $F_{0}(\infty) \subset X_{g}(\infty)$, which is impossible. We got $\operatorname{Min}(g)=\gamma_{0}$. We finished Case 4.

We are left with those $g$ which are diagonal. Suppose $e \neq g=\operatorname{diag}(a, b, c) \in$ $\mathrm{SL}(3, \mathbb{R}) . \quad g$ is a semi-simple isometry and the flat $F_{0}$ is $g$-invariant, so that $A_{0} \subset X_{g}(\infty)$. 
Set

$$
N=\sqrt{(\log |a|)^{2}+(\log |b|)^{2}+(\log |c|)^{2}}
$$

and define a unit length element $u_{g} \in T_{e} F_{0}$ by

$$
u_{g}:=\frac{1}{N} \operatorname{diag}(\log |a|, \log |b|, \log |c|) .
$$

Let $\gamma_{g}$ be the bi-infinite geodesic in $F_{0}$ through $e$ defined by

$$
\gamma_{g}(t):=\exp \left(t u_{g}\right), \quad t \in \mathbb{R} .
$$

This is of unit speed. Computation shows that $\gamma_{g}$ is $g$-invariant, therefore it is an axis. $|g|=d(e, g(e))=d\left(e, g^{t} g\right) . g^{t} g=\operatorname{diag}\left(a^{2}, b^{2}, c^{2}\right)=\exp \left(2 N u_{g}\right)=\gamma_{g}(2 N)$. Since $\gamma_{g}$ has unit speed, $|g|=2 N$.

There are two cases: $\gamma_{g}$ is regular (Case 5) or singular (Case 6). We already know that $g$ is hyperbolic and calculated $|g|$.

\section{Case 5.}

(a) Since $F_{0}$ is invariant by $g, A_{0}=F_{0}(\infty) \subset X_{g}(\infty)$. Let $v \in X_{g}(\infty)$. Then there is a flat $F$ with $\gamma_{g} \subset F$ and $v \in F(\infty)$. Indeed, if $\gamma$ is a bi-infinite geodesic through $e$ with $\gamma(\infty)=v$, then since $g(v)=v, \gamma$ and $\gamma_{g}$ is on some flat.

Since $\gamma_{g}$ is a regular geodesic, it is contained in only one flat, so that $F=F_{0}$. We get $v \in F_{0}(\infty)=A_{0}$.

(b) Since $F_{0}$ is $g$-invariant, $F_{0} \subset \operatorname{Min}(g)$. $\operatorname{Min}(g)$ consists of axes of $g$. Let $\gamma$ be an axis different from $\gamma_{g}$. Then there is a flat strip between them, so that there is indeed a flat, $F$, containing both of them because it is in a symmetric space. Since $\gamma_{g}$ is regular, we have $F=F_{0}$, so that $\gamma$ is in $F_{0}$.

Case 6.

(a) As in Case 5, $A_{0} \subset X_{g}(\infty)$. Since $g$ commutes with any element in $R$, $X_{g}(\infty)$ is $R$-invariant, so that $R \cdot A_{0} \subset X_{g}(\infty) . R \cdot A_{0}=P\left(\gamma_{0}\right)(\infty)$ implies that $P\left(\gamma_{0}\right)(\infty) \subset X_{g}(\infty)$. To see the other inclusion, let $v \in X_{g}(\infty)$. Then there is a flat, $F$, such that $\gamma_{0} \subset F$ and $v \in F(\infty)$ (cf. (a) in Case 5). By definition, $F \in \widetilde{F}_{0}$, so that $F \subset P\left(\gamma_{0}\right)$. We get $v \in P\left(\gamma_{0}\right)(\infty)$.

(b) In this case, $\gamma_{g}=\gamma_{0} . \quad F_{0}$ is $g$-invariant, so that $F_{0} \subset \operatorname{Min}(g)$. Since $g$ commutes with any element in $R, \operatorname{Min}(g)$ is $R$-invariant, so that $R \cdot F_{0} \subset \operatorname{Min}(g)$. Because $R \cdot F_{0}=P\left(\gamma_{0}\right), P\left(\gamma_{0}\right) \subset \operatorname{Min}(g)$. On the other hand, since $P\left(\gamma_{0}\right)$ is the union of all geodesics parallel to $\gamma_{0}, \operatorname{Min}(g) \subset P\left(\gamma_{0}\right)$, therefore $\operatorname{Min}(g)=P\left(\gamma_{0}\right)$. Case 6 is done.

To finish the proof, we show $|g|=2 \sqrt{6} \log |a|$ for $g$ in Case 2. It is easy to see that $g$ is conjugate in $\operatorname{SL}(3, \mathbb{R})$ to the matrix, $h=\left(\begin{array}{ccc}a & 1 & 0 \\ 0 & a & 0 \\ 0 & 0 & 1 / a^{2}\end{array}\right)$, so that it suffices to show $|h|=2 \sqrt{6} \log |a|$. 
Although $\gamma_{0}$ is not $h$-invariant, $h$ fixes $\gamma_{0}(\infty)=v_{2}, \gamma_{0}(-\infty)=v_{5}$ because $h \in G_{2} \cap G_{5}$, so that $h$ leaves not only the subset $P\left(\gamma_{0}\right)$, but also its product structure $P\left(\gamma_{0}\right)=\mathbb{H}^{2} \times \mathbb{R}$ invariant.

The restriction of $h$ to $P=P\left(\gamma_{0}\right),\left.h\right|_{P}$, is also parabolic. Since $P$ is convex in $X$ and $h$-invariant, $|h|_{P}|=| h \mid$, so that we compute $|h|_{P}|. h|_{P}$ acts on $P=\mathbb{H}^{2} \times \mathbb{R}$ by a product of isometries: a parabolic isometry on $\mathbb{H}^{2}$, denoted by $\left.h\right|_{\mathbb{H}^{2}}$, and a translation on $\mathbb{R}$, denoted by $\left.h\right|_{\mathbb{R}}$. Since $|h|_{\mathbb{H}^{2}} \mid=0$, we have $\left.|h|_{P}|=| h\right|_{\mathbb{R}} \mid$.

Consider the following matrix in $\operatorname{SL}(3, \mathbb{R}), k=\left(\begin{array}{ccc}1 & -1 / a & 0 \\ 0 & 1 & 0 \\ 0 & 0 & 1\end{array}\right)$. This is also a parabolic isometry, which leaves $P\left(\gamma_{0}\right)$ invariant such that it acts on it as a product of isometries of $\mathbb{H}^{2}$ and $\mathbb{R}$. The action of $k$ on $\mathbb{R}$ is trivial since the $(3,3)$-entry of $k$ is 1 . This is because one can show by computation that the geodesic from $e \in X$ to $k(e)$ is perpendicular to $\gamma_{0}$ at $e$, so that $k(e) \in \mathbb{H}^{2}$ in $\mathbb{H}^{2} \times \mathbb{R}$. Or, one may use the fact that $P\left(\gamma_{0}\right)$ is the union of matrices of the following form in $P(3, \mathbb{R})_{1} ;\left(\begin{array}{lll}* & * & 0 \\ * & * & 0 \\ 0 & 0 & *\end{array}\right)$, where the set of top-left $(2 \times 2)$-matrices corresponds to $\mathbb{H}^{2}$ and the $(3,3)$-entries, which are positive numbers, (by taking $\log$ ) correspond to $\mathbb{R}$ in the product decomposition $P\left(\gamma_{0}\right)=\mathbb{H}^{2} \times \mathbb{R}$. By the definition of the action, $k$ acts trivially on the second factor. Therefore, $\left.|h k|_{P}|=| h\right|_{P} \mid$. By the same reason as $h,|h k|_{P}|=| h k \mid$. By computation, $h k=\operatorname{diag}\left(a, a, 1 / a^{2}\right)$, which is hyperbolic. We have just computed that $\left|\operatorname{diag}\left(a, a, 1 / a^{2}\right)\right|=2 \sqrt{6} \log |a|$. To summarize,

$$
|g|=|h|=\left.|h|_{P}|=| h k\right|_{P}|=| h k|=2 \sqrt{6} \log | a \mid .
$$

We finished the proof.

\section{References}

[B] W. Ballmann, Lectures on spaces of nonpositive curvature. DMV-Seminar 25, Birkhäuser, Basel 1995. Zbl 0834.53003 MR 1377265

[BGS] W. Ballmann, M. Gromov, and V. Schroeder, Manifolds of nonpositive curvature. Progr. Math. 61, Birkhäuser, Boston, MA, 1985. Zbl 0591.53001 MR 0823981

[BH] M. R. Bridson and A. Haefliger, Metric spaces of non-positive curvature. Grundlehren Math. Wiss. 319, Springer-Verlag, Berlin 1999. Zbl 0988.53001 MR 1744486

[BBI] D. Burago, Yu. Burago, and S. Ivanov, A course in metric geometry. Grad. Stud. in Math. 33, Amer. Math. Soc., Providence, RI, 2001. Zbl 0981.51016 MR 1835418

[Bu] S. V. Buyalo, Geodesics in Hadamard spaces. St. Petersburg Math. J. 10 (1999), 293-313. Zbl 0918.53018 MR 1629391

[E] P. Eberlein, Geometry of nonpositively curved manifolds, Chicago Lectures in Mathematics, The University of Chicago Press, Chicago, IL, 1996. Zbl 0883.53003 MR 1441541 
Vol. 81 (2006)

[F] V. V. Fedorchuk, The fundamentals of dimension theory. In General Topology I, Encyclopaedia Math. Sci. 17, Springer-Verlag, Berlin 1990, 91-192. Zbl 0780.54034 MR 1077251

[FSY] K. Fujiwara, T. Shioya, and S. Yamagata, Parabolic isometries of CAT(0) spaces and CAT(0)-dimension. Algebr. Geom. Topol. 4 (2004), 861-892 (electronic). Zbl 02139842 MR 2100684

[J] J. Jost, Nonlinear Dirichlet forms. In New directions in Dirichlet forms, AMS/IP Stud. Adv. Math. 8, Amer. Math. Soc., Providence, RI, 1998, 1-47. Zbl 0914.31006 MR 1652278

[K] B. Kleiner, The local structure of length spaces with curvature bounded above. Math. Z. 231 (1999), 409-456. Zbl 0940.53024 MR 1704987

[LS1] U. Lang and V. Schroeder, Kirszbraun's theorem and metric spaces of bounded curvature. Geom. Funct. Anal. 7 (1997), 535-560. Zbl 0891.53046 MR 1466337

[LS2] - Jung's theorem for Alexandrov spaces of curvature bounded above. Ann. Global Anal. Geom. 15 (1997), 263-275. Zbl 0974.53028 MR 1456512

[L] A. Lytchak, Rigidity of spherical buildings and joins. Geom. Funct. Anal. 15 (2005), 720-752.

[M] U. Mayer, Gradient flows on nonpositively curved metric spaces and harmonic maps. Comm. Anal. Geom. 6 (1998), 199-253. Zbl 0914.58008 MR 1651416

Received September 9, 2004

Koji Fujiwara, Mathematical Institute, Tohoku University, Aoba, Sendai, Miyagi, 980-8578, Japan

E-mail: fujiwara@math.tohoku.ac.jp

Koichi Nagano, Mathematical Institute, Tohoku University, Aoba, Sendai, Miyagi, 980-8578, Japan

E-mail: nagano@math.tohoku.ac.jp

Takashi Shioya, Mathematical Institute, Tohoku University, Aoba, Sendai, Miyagi, 980-8578, Japan

E-mail: shioya@math.tohoku.ac.jp 